\title{
Non-symmetrical allenyl azines and their transformations leading to new heterocyclic skeletons
}

\author{
Juraj Galeta, Stanislav Man, and Milan Potáček* \\ Department of Chemistry, Faculty of Science, Masaryk University, Kotlářská 2, 61137 Brno, \\ Czech Republic \\ E-mail:potacek@,chemi.muni.cz
}

The paper is dedicated to Professor Henk van der Plas on the occasion of his 80th anniversary

\begin{abstract}
The preparation of new non-symmetrical allenyl azines $\mathbf{5}$ with aliphatic and alicyclic substituents and their reactions are described. When aldazines $\mathbf{5}$ were refluxed in xylene formation of bicyclic products 7 was observed. Compounds 7 appeared as derivatives of isowithasomnine. Combined intra-intermolecular criss-cross cycloadditions of azines $\mathbf{5}$ afforded heterocyclic products 8 and 9 containing three fused five-membered rings. Structures of all new compounds were elucidated by ${ }^{1} \mathrm{H}$ and ${ }^{13} \mathrm{C}$ NMR, IR and MS measurements and in some cases by X-ray structure analysis.
\end{abstract}

Keywords: Allene, non-symmetrical azines, 1,3-dipolar cycloadditions, cyclizations, intraintermolecular criss-cross cycloaddition, isowithasomnine

\section{Introduction}

For a long time, the chemistry of the allenyl synthon was neglected in heterocyclic chemistry. Only in the past decade has the number of published papers increased and a survey of its chemical reactivity appeared. ${ }^{1}$ However, our laboratory belongs to the working places where the chemistry of allene derivatives was investigated. We have investigated mostly derivatives of homoallenyl aldehyde 1, because we have developed its improved preparation procedure (Scheme 1). ${ }^{2}$ Among all the derivatives symmetrical azines play important role as educts of intramolecular criss-cross cycloadditions. ${ }^{3}$ Fused tetracyclic compounds, formed in this fashion, as products of two successive 1,3-dipolar cycloadditions, ${ }^{4}$ have been the source of interesting information about the mechanism of their formation and possible further transformations. ${ }^{5}$ 


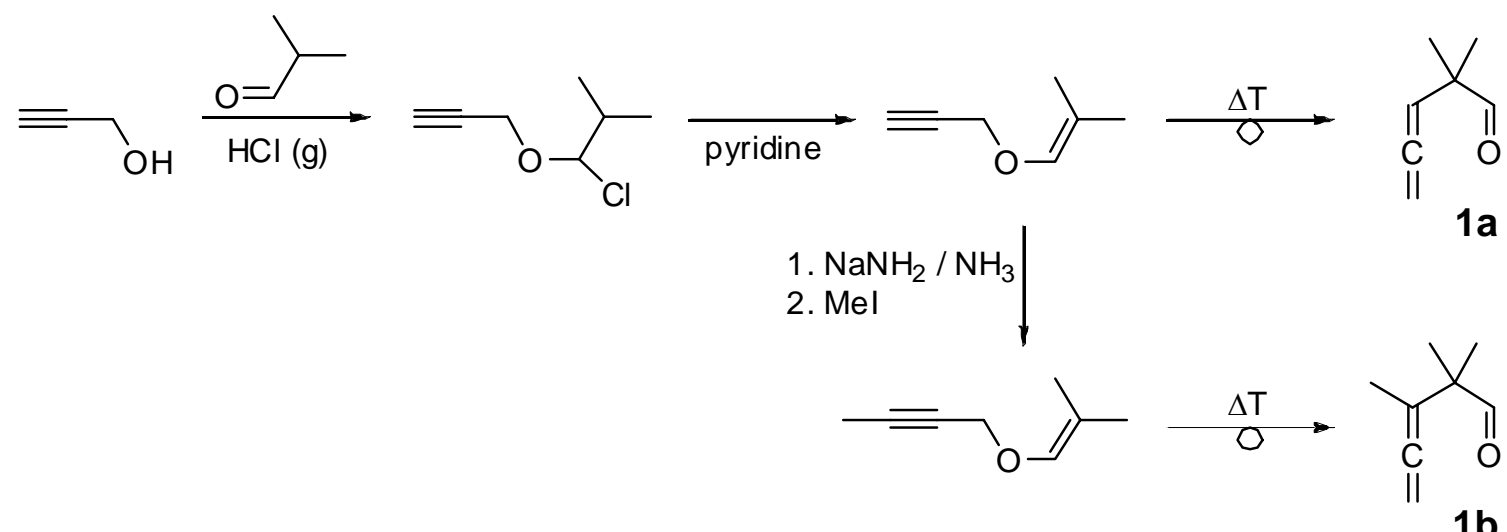

Scheme 1. Preparation of homoallenyl aldehydes $\mathbf{1 a}$ and $\mathbf{1 b}$.

Homoallenyl oxime prepared from compound $\mathbf{1}$ appeared to be a very fruitful molecule undergoing many further useful transformations via a stable nitrone, which underwent further reactions either with preservation of the dipole or with its participation in a 1,3-dipolar cycloaddition and formation of new skeletons. ${ }^{6}$ On the other hand non-symmetrical allenyl azines 5, especially those with an aliphatic and alicyclic substituents are not known. Therefore we concentrated on the preparation of this type of compounds, and then to investigation of their transformations.

The method published by Zwierzak enabled a comfortable preparation of non-symmetrical azines. ${ }^{7}$ In this method the first step consists of one nitrogen of hydrazine molecule protection by the reaction with diethyl phosphite forming diethyl hydrazidophosphate $\mathbf{2}$. The intermediate $\mathbf{2}$ was reacted the usual way with one carbonyl compound to form the protected hydrazones $\mathbf{3}$ or $\mathbf{4}$. Treatment of the hydrazone in the presence of sodium hydride in dry ether with another carbonyl compound yielded the non-symmetrical azine 5 (Scheme 2).

Some of these new non-symmetrical allenyl azines 5 were reacted with different dipolarophiles by thermally initiated combined intra-intermolecular criss-cross cycloaddition (CCC) (Scheme 5). The CCCs reactions may be classified as a special type of $[3+2]$ cycloaddition, ${ }^{8}$ or 1,3-dipolar cycloaddition. These reactions afforded new heterocycles $\mathbf{8}$ with three fused five-membered rings. The formation of criss-cross products was first time explained by Huisgen ${ }^{4}$ in 1963 as a succession of two successive 1,3-dipolar cycloadditions. This explanation was proven in 1973 when a stable 1,3-dipole was identified by X-ray crystallographic analysis. ${ }^{9}$ 


\section{Results and Discussion}

\section{Preparation of non-symmetrical homoallenyl azines}

The synthesis of homoallenyl aldehydes 1a and $\mathbf{1 b}$ was carried out according the described procedure (Scheme 1). ${ }^{2}$ The synthesis of the non-symmetrical allenyl azines $\mathbf{5}$ from the allenyl aldehyde $\mathbf{1}$ can be performed by two different pathways (A and B) as shown at the following Scheme 2. In most of the cases the preparation was carried out via aliphatic or alicyclic protected hydrazone 3 from protected hydrazidate 2 and by reaction with homoallenyl aldehyde $\mathbf{1}$. The preferred way is (A) because in the first step of reaction the less expensive aldehyde or ketone is used. The other path (B) consisting of reaction of homoallenyl aldehyde $\mathbf{1}$ with protected hydrazidate 2 led to the protected homoallenyl hydrazone 4. Subsequent reaction with the corresponding aliphatic aldehyde or ketone afforded the non-symmetrical azine 5. Although both pathways could be used for azines $\mathbf{5}$, azines $\mathbf{5 a}, \mathbf{b}$ (where R' and R" $=\mathrm{H}$ ) could only be prepared according to path $\mathbf{B}$. When preparation was attempted according to path $\mathbf{A}$, tar mixtures were isolated.

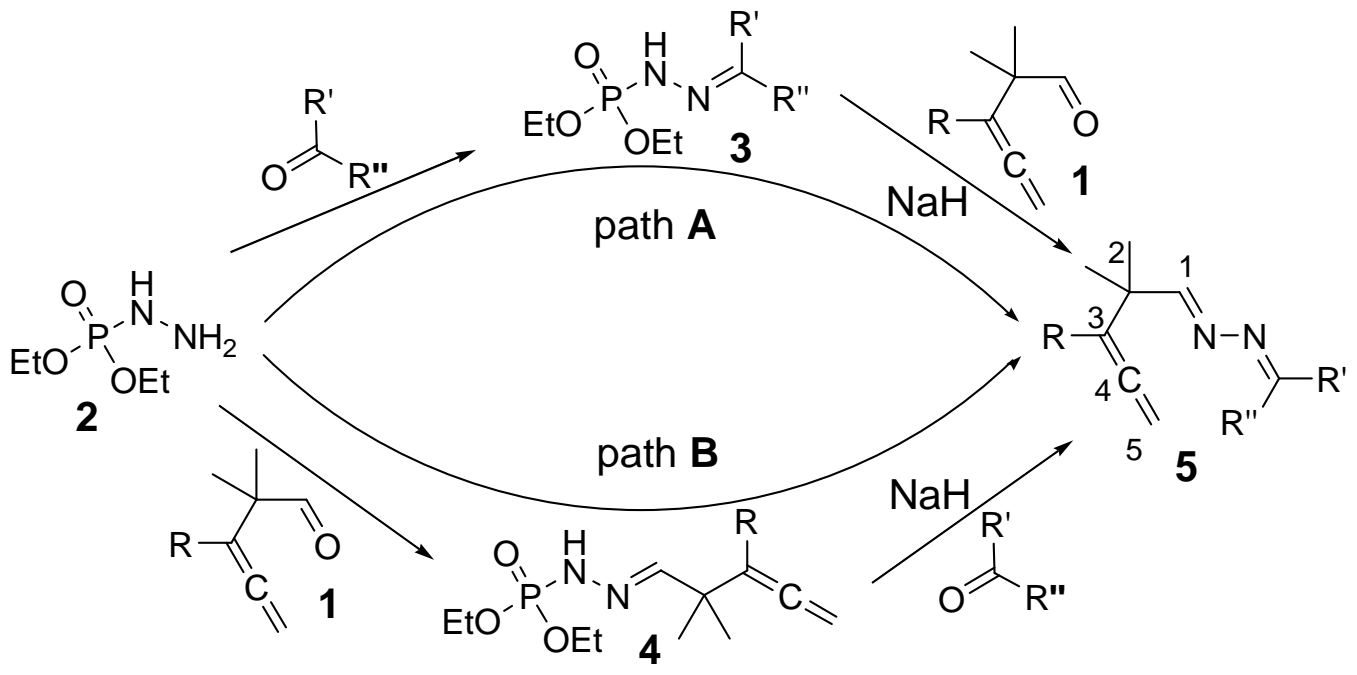

Scheme 2. Preparation of allenyl azines 5.

Monitoring of azine 5 formation (Table 1) was carried out by TLC and interestingly also by the odor of reaction mixture because of the typical and strong smell of forming compound $\mathbf{5}$. Another important attribute of azines $\mathbf{5}$ with aliphatic or alicyclic substitution is their rather high stability at room temperature (no decomposition was observed within several weeks). 
Table 1. Non-symmetrical allenyl azines 5a-n and their yields

\begin{tabular}{cccccc}
\hline Compound $\mathbf{5}$ & $\mathrm{R}$ & $\mathrm{R}^{\prime}$ & $\mathrm{R}^{\prime \prime}$ & yield [\%] & time $[\mathrm{h}]$ \\
\hline $\mathbf{a}$ & $\mathrm{H}$ & $\mathrm{H}$ & $\mathrm{H}$ & 89 & 1 \\
$\mathbf{b}$ & $\mathrm{Me}$ & $\mathrm{H}$ & $\mathrm{H}$ & 54 & 1.2 \\
$\mathbf{c}$ & $\mathrm{H}$ & $\mathrm{Me}$ & $\mathrm{H}$ & 61 & 3 \\
$\mathbf{d}$ & $\mathrm{Me}$ & $\mathrm{Me}$ & $\mathrm{H}$ & 70 & 3 \\
$\mathbf{e}$ & $\mathrm{H}$ & $\mathrm{Et}$ & $\mathrm{H}$ & 79 & 2 \\
$\mathbf{f}$ & $\mathrm{Me}$ & $\mathrm{Et}$ & $\mathrm{H}$ & 54 & 2.5 \\
$\mathbf{g}$ & $\mathrm{H}$ & $i-\mathrm{Pr}$ & $\mathrm{H}$ & 80 & 2 \\
$\mathbf{h}$ & $\mathrm{Me}$ & $i-\mathrm{Pr}$ & $\mathrm{H}$ & 78 & 3.5 \\
$\mathbf{i}$ & $\mathrm{H}$ & $\mathrm{CH}=\mathrm{CH}-\mathrm{Me}$ & $\mathrm{H}$ & 89 & 2 \\
$\mathbf{j}$ & $\mathrm{Me}$ & $\mathrm{CH}=\mathrm{CH}-\mathrm{Me}^{-}$ & $\mathrm{H}$ & 56 & 3 \\
$\mathbf{k}$ & $\mathrm{H}$ & $-\left(\mathrm{CH}_{2}\right)_{4^{-}}$ & & 84 & 1.5 \\
$\mathbf{l}$ & $\mathrm{Me}$ & $-\left(\mathrm{CH}_{2}\right)_{4^{-}}$ & & 81 & 1.5 \\
$\mathbf{m}$ & $\mathrm{H}$ & $-\left(\mathrm{CH}_{2}\right)_{5^{-}}$ & 82 & 1.5 \\
$\mathbf{n}$ & $\mathrm{Me}$ & $-\left(\mathrm{CH}_{2}\right)_{5^{-}}$ & 80 & 1 \\
\hline
\end{tabular}

In the IR spectra, products 5 were characterized by strong $\mathrm{C}=\mathrm{N}$ vibration (between 1606 $\left.1659 \mathrm{~cm}^{-1}\right)$ and strong $\mathrm{C}=\mathrm{C}=\mathrm{C}$ vibration $\left(\sim 1954 \mathrm{~cm}^{-1}\right)$. The molecular weights of all the products were confirmed by MS by the presence of their molecular ion-peaks.

Absence of a stereogenic centre is typical for all these compounds 5. Therefore, we can observe only one signal for both of the methyl groups at $\mathrm{C} 2$. Characteristic for ${ }^{1} \mathrm{H}$ NMR spectra of compounds with allenyl skeleton $\mathrm{C}_{2}=\mathrm{C}=\mathrm{CR}$ - is a long distance interaction between protons with coupling constants ${ }^{4} J_{\mathrm{H}, \mathrm{H}} \sim 6.6 \mathrm{~Hz}$ for $\mathrm{R}=\mathrm{H}$ and ${ }^{5} J_{\mathrm{H}, \mathrm{H}} \sim 3.0 \mathrm{~Hz}$ for $\mathrm{R}=\mathrm{Me}$. A similar situation exists with both methyl groups in ${ }^{13} \mathrm{C}$ NMR spectrum.

\section{Reactions of azines 5}

In the case that homoallenyl aldazines $\mathbf{5}$ are refluxed in dry xylene, formation of bicyclic derivatives 7 was observed (Scheme 3, Table 3).
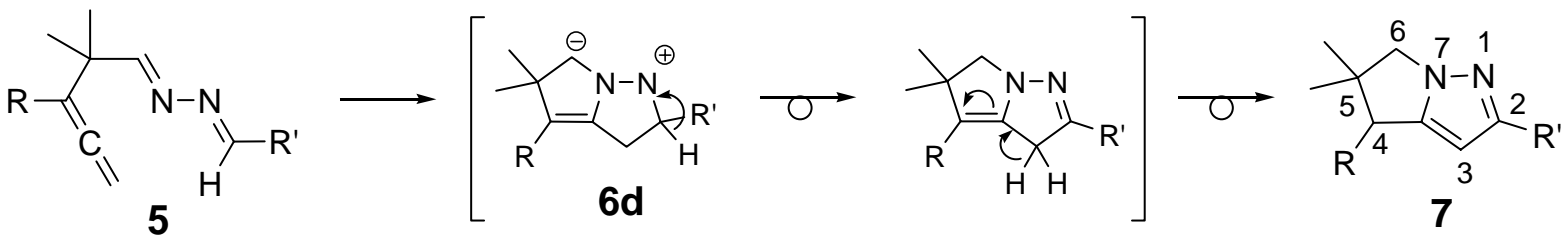

Scheme 3. Formation of bicyclic compounds 7. 
Table 3. Characteristics of bicyclic products 7a-c

\begin{tabular}{cccccc}
\hline Compound & $\mathrm{R}$ & $\mathrm{R}$ & $\begin{array}{c}\text { Yield } \\
{[\%]}\end{array}$ & Time $[\mathrm{h}]$ & Purification \\
\hline $\mathbf{7 a}$ & $\mathrm{Me}$ & $\mathrm{H}$ & 35 & 5 & $\operatorname{AcOEt} / \mathrm{PE}(1: 3)$ \\
$\mathbf{7 b}$ & $\mathrm{Me}$ & $\mathrm{Me}$ & $\sim 50$ & 5 & $\mathrm{Et}_{2} \mathrm{O}$ \\
$\mathbf{7 c}$ & $\mathrm{Me}$ & $i-\mathrm{Pr}$ & 94 & 6 & $\mathrm{Et}_{2} \mathrm{O} / \mathrm{PE}(1: 1)$ \\
\hline
\end{tabular}

Formation of such structures has already been observed when aromatic homoallenyl azines were heated with low reactive dipolarophiles. ${ }^{10,11}$ Compounds 7 prepared in this way are derivatives of isowithasomnine. Products 7 contain an aliphatic chain instead of phenyl in isowithasomnine in position 2. Isowithasomnine differs from withasomnine, a plant alkaloid known as Indian Gin-Seng, by position of the phenyl and was isolated from plants Withania somnifera Dun, ${ }^{12}$ Elytraria acaulis ${ }^{13}$ and Newbouldia laevis. ${ }^{14}$ It has anesthetic and narcotic properties ${ }^{15}$ as well as being an inhibitor of malignant growth. ${ }^{16}$ Our reaction afforded reasonable yields only in case the applied azine is aldazine. Otherwise a rather complicated mixture of decomposition products is obtained.

When considering the reaction mechanism, the initial step of the process is an intramolecular attack (probably the rate determining step) via formation of dipolar intermediate 6, which may be written in four resonance structures 6a-d (Scheme 4). Formation of such a product was also predicted in our previous study by ab initio calculations. ${ }^{17}$ The intermediate 6 then undergoes protons shifts affording compound 7 (Scheme 3).

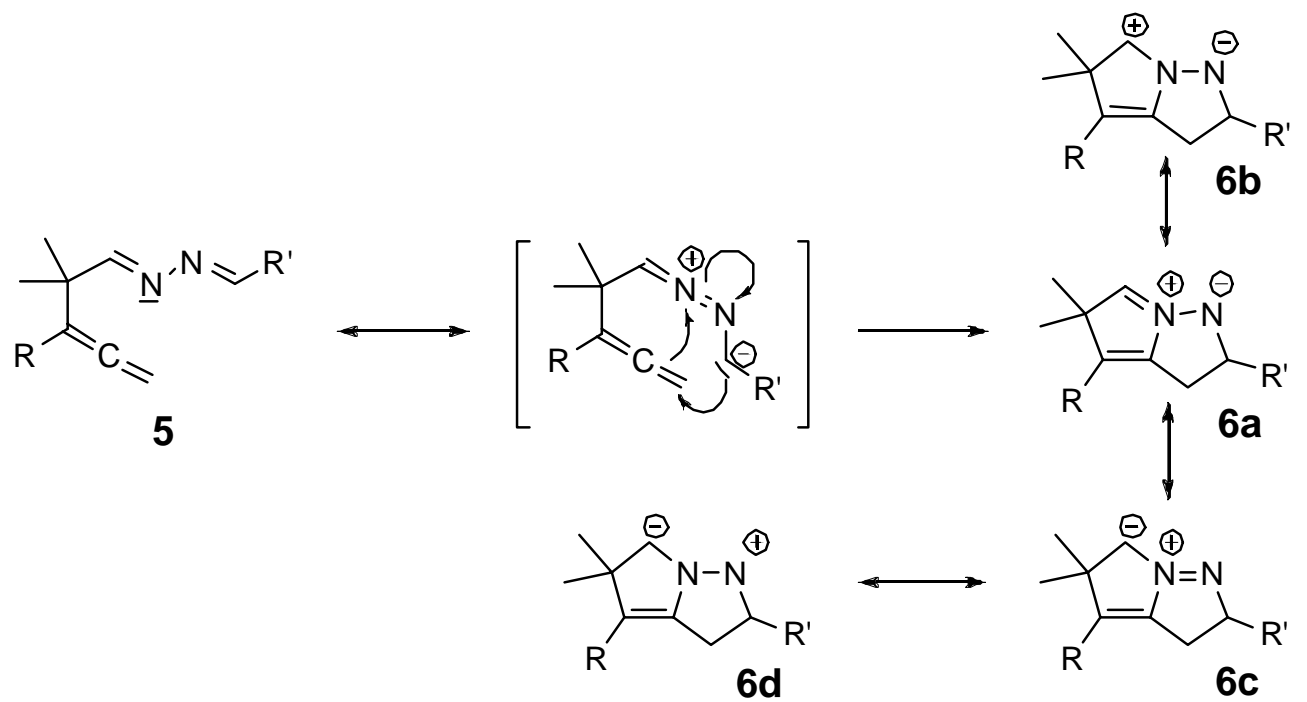

Scheme 4. Generation of 1,3-dipole 6a-d, as a reactive intermediate, in dry boiling xylene.

In case we add into reaction mixture a reactive dipolarophile the in situ formed dipole reacts to product containing three fused five-membered heterocyclic rings (Scheme 5). 

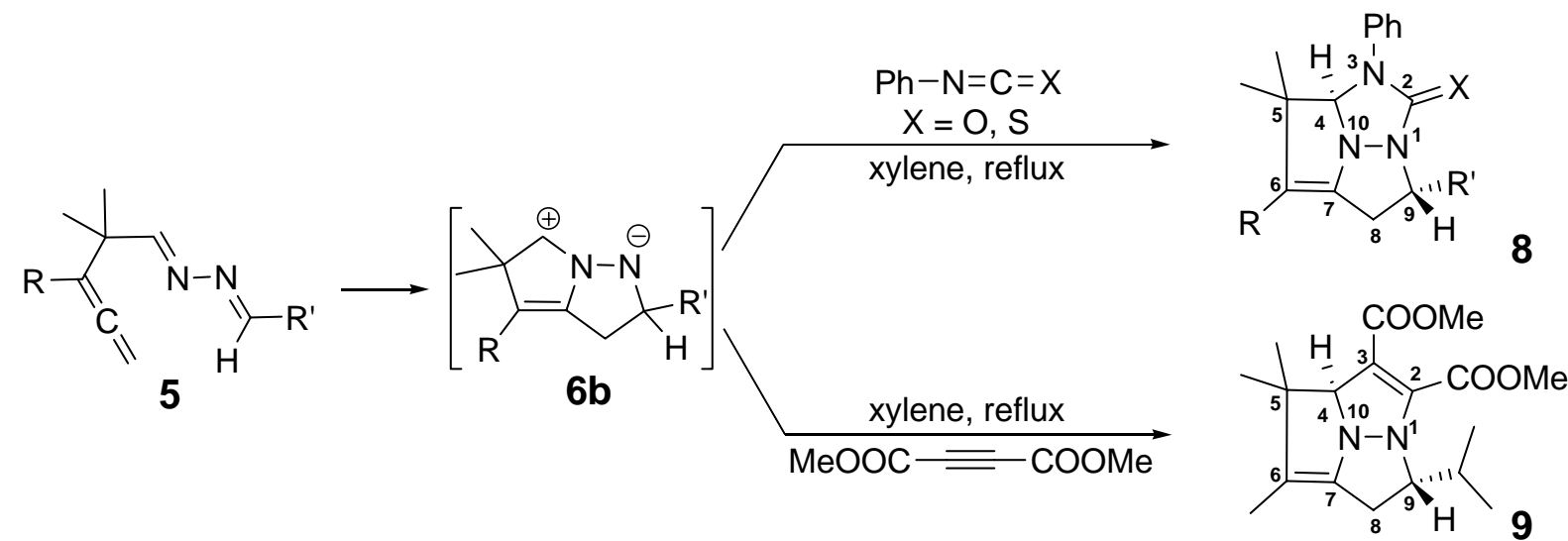

Scheme 5. Intermolecular criss-cross cycloaddition of 1,3-dipole $\mathbf{6 b}$ with dipolarophiles.

This way in so called combined intra-intermolecular criss-cross cycloaddition were prepared new heterocyclic compounds 8 and $\mathbf{9}$ (Table 4) with three fused five-membered rings (Scheme 5).

Table 4. Overview of prepared compounds 8 and 9 and information for their preparation and purification

\begin{tabular}{|c|c|c|c|c|c|c|c|c|}
\hline Compound & $\mathrm{R}$ & $R^{6}$ & dipolarophile & $\begin{array}{c}\text { yield } \\
{[\%]}\end{array}$ & $\begin{array}{l}\text { time } \\
{[\mathrm{h}]}\end{array}$ & purification & $\begin{array}{c}\text { ratio } \\
\text { azine/dipolar. } \\
{[\mathrm{mmol}]}\end{array}$ & $\begin{array}{l}\text { solvent } \\
{[\mathrm{ml}]}\end{array}$ \\
\hline $8 \mathbf{a}$ & $\mathrm{H}$ & $\mathrm{H}$ & $\mathrm{PhNCO}$ & 50 & 5 & AcOEt/PE (1:4) & $2 / 2.4$ & 60 \\
\hline $8 b$ & $\mathrm{Me}$ & $\mathrm{H}$ & $\mathrm{PhNCO}$ & 61 & 3 & $\begin{array}{c}\text { EtOH/Hexan } \\
(\sim 2: 1)\end{array}$ & $1 / 1.2$ & 30 \\
\hline $8 c$ & $\mathrm{H}$ & $\mathrm{Me}$ & $\mathrm{PhNCO}$ & 40 & 6 & AcOEt/PE (1:4) & $2.6 / 2.8$ & 80 \\
\hline 8d & $\mathrm{Me}$ & $\mathrm{Me}$ & $\mathrm{PhNCO}$ & 93 & 5 & $\mathrm{AcOEt} / \mathrm{PE}(1: 3)$ & $1 / 1.2$ & 30 \\
\hline $8 e$ & $\mathrm{H}$ & $\mathrm{Et}$ & $\mathrm{PhNCO}$ & 41 & 5 & $\operatorname{AcOEt} / \mathrm{PE}(1: 6)$ & $3.3 / 3.5$ & 100 \\
\hline $8 f$ & $\mathrm{Me}$ & Et & $\mathrm{PhNCO}$ & 45 & 8 & $\begin{array}{c}\mathrm{Et}_{2} \mathrm{O} / \mathrm{PE}(\sim 1: 1) \\
-10^{\circ} \mathrm{C}\end{array}$ & $1.5 / 1.6$ & 45 \\
\hline $8 g$ & $\mathrm{H}$ & $\mathrm{H}$ & BnNCS & 40 & 3 & $\mathrm{AcOEt} / \mathrm{PE}(1: 4)$ & $3 / 3$ & 30 \\
\hline $8 h$ & $\mathrm{H}$ & $\mathrm{H}$ & $\mathrm{PhNCS}$ & 39 & 4 & $\mathrm{PE} / \mathrm{Et}_{2} \mathrm{O}(\sim 5: 1)$ & $2 / 2$ & 20 \\
\hline $8 \mathbf{i}$ & $\mathrm{Me}$ & $\mathrm{H}$ & PhNCS & 78 & 3 & $\begin{array}{c}\text { EtOH/Hexane } \\
(\sim 2: 1)\end{array}$ & $1 / 1$ & 10 \\
\hline 9 & $\mathrm{Me}$ & $i-\operatorname{Pr}$ & DAD $^{*}$ & 90 & 5 & $\mathrm{Et}_{2} \mathrm{O} / \mathrm{PE}(8: 2)$ & $0.6 / 1.2$ & 25 \\
\hline
\end{tabular}

* $\mathrm{DAD}=$ dimethylacetylene dicarboxylate; $\mathrm{PE}=$ petroleum ether 
These structures were elucidated by NMR spectroscopic measurements. In contrast to allenyl azines 5, compounds 8 contain stereogenic centres. Therefore the methyl groups at the C5 are diastereotopic and we can find their signals as two distinct entities differing by about $0.5 \mathrm{ppm}$ in ${ }^{1} \mathrm{H}$ NMR and by $3-5 \mathrm{ppm}$ in ${ }^{13} \mathrm{C}$ NMR spectra. The other signals are presented in the Experimental section. For approval of the structure X-ray diffraction analysis was carried out. The measured compounds appeared as a racemate of one diastereoisomer only (Figure 1).
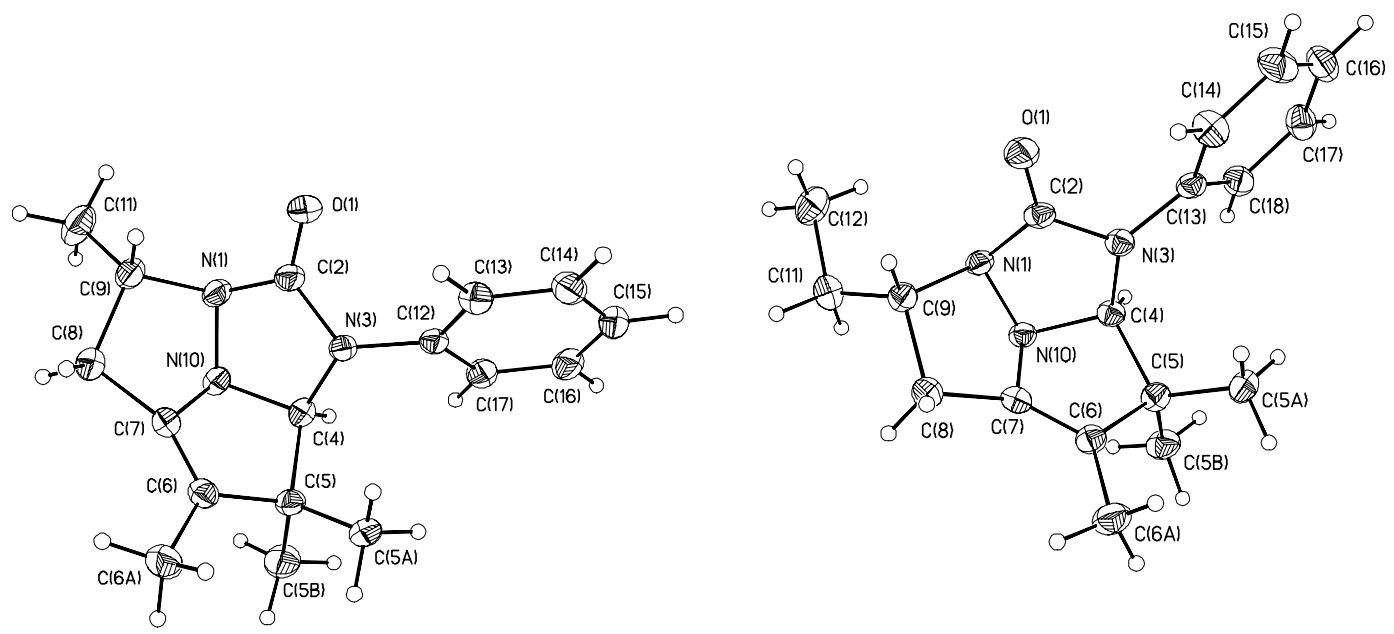

Figure 1. X-ray structure of compounds $\mathbf{8 d}$ and $\mathbf{8 f}$.

Because of the sterically demanding group $\mathrm{R}^{\prime}$ the dipolarophile is approaching the molecule of 1,3-dipole from one side only and therefore one diastereoisomer is formed.

\section{Conclusions}

We have succeeded in synthesising new non-symmetrical homoallenyl azines (aldazines 5a-j and aldoketazines 5k-n) in reasonable yields and fully characterise them. When azines $\mathbf{5}$ were heated without any dipolarophile products 7 belonging to derivatives of isowithasomnine were isolated. Further azines 5 in the presence of reactive dipolarophiles under thermally initiated reaction underwent intra-intermolecular criss-cross cycloaddition via formation of 1,3-dipole affording new heterocyclic skeletons $\mathbf{8}$ and $\mathbf{9}$, respectively, consisting from three fused five-membered rings.

\section{Experimental Section}

General Procedures. Melting points were determined on a Kofler hot-stage apparatus and are uncorrected. FT-IR spectra were measured with GENESIS ATI (Unicam) spectrometer. ${ }^{1} \mathrm{H}$ and 
${ }^{13} \mathrm{C}$ NMR spectra were recorded on Bruker AVANCE 300 spectrometer in $\mathrm{CDCl}_{3}$. Tetramethylsilane $(\delta=0.00 \mathrm{ppm})$ or $\mathrm{CHCl}_{3}(\delta=7.27 \mathrm{ppm})$ served as an internal standards for ${ }^{1} \mathrm{H}$ NMR (300 MHz) and $\mathrm{CDCl}_{3}$ as an internal standard $(\delta=77.23 \mathrm{ppm})$ for ${ }^{13} \mathrm{C}$ NMR $(75.5 \mathrm{MHz})$. Coupling constants are reported in Hertz [Hz]. MS data were obtained on a FISONS INSTRUMENTS TRIO 1000 spectrometer with EI ionization (30 eV) and GC-MS on SHIMADZU GCMS-QP2010 in EI mode (30 eV). HRMS were taken at Waters-Micromas, QTof Micro (ESSI positive).

X-Ray diffraction data were collected with a Kuma KM-4 four-circle CCD diffractometer and corrected for Lorentz and polarization effects. The structure was solved by direct methods and refined by using a SHELXTL program package. ${ }^{19}$ The hydrogen atoms were placed in calculated idealized positions. Liquid chromatography was carried out on Horizon HPFC System (Biotage, Inc.) with Biotage $\mathrm{Si} 12+\mathrm{M}$ and $25+\mathrm{M}$ columns. Xylene (mixture of isomers), benzene and diethylether were dried and distilled from sodium/benzophenone and stored under argon. All reactions were carried out under a dry argon atmosphere and monitored by TLC on plates coated with Merck silica gel $\mathrm{F}_{254}$.

\section{General procedure for the preparation of $5 a-b$ and $5 c-n$}

A mixture of allenyl hydrazone $4(10 \mathrm{mmol})$ and paraformaldehyde $\left(20 \mathrm{mmol}\right.$, equiv. $\left.\mathrm{CH}_{2} \mathrm{O}\right)$ in benzene $(30 \mathrm{ml})$ was slowly added to a suspension of $\mathrm{NaH}(0.36 \mathrm{~g}, 15 \mathrm{mmol})$ in diethylether $(30$ $\mathrm{ml})$ at $5-10{ }^{\circ} \mathrm{C}$. Then the reaction mixture was left stirring at room temperature. The solution was filtered and the residue washed with diethylether $(3 \times 20 \mathrm{ml})$. The combined organic phases were concentrated in vacuo and the crude product was purified by vacuum distillation using Kugelrohr apparatus to give colorless or yellowish liquid.

The procedure for the preparation of 5c-n differs in used amounts of reactants only. The reaction mixture contains $10.5 \mathrm{mmol}$ of protected hydrazone 3 and $10 \mathrm{mmol}$ of allenyl aldehyde $\mathbf{1}$.

(2,2-Dimethylpenta-3,4-dien-1-ylidene) formaldehyde hydrazone (5a). IR (film) $v_{\max } / \mathrm{cm}^{-1}$ 845, 972, 1196, 1362, 1383, 1464, $1606(\mathrm{C}=\mathrm{N}), 1637(\mathrm{C}=\mathrm{N}), 1954(=\mathrm{C}=), 2866,2927,2966$. MS m/z (\%) $137\left(\mathrm{M}^{+}+1 ; 18\right), 121$ (100), 108 (73), 94 (54), 79 (74), 67 (58), 53 (46), 41 (73). ${ }^{1} \mathrm{H}$ NMR $\delta 1.26\left(\mathrm{~s}, 6 \mathrm{H}, \mathrm{H}_{3} \mathrm{C}-\mathrm{C}-\mathrm{CH}_{3}\right), 4.82\left(\mathrm{~d},{ }^{4} J_{\mathrm{H}, \mathrm{H}}=6.6 ; 2 \mathrm{H},=\mathrm{CH}_{2}\right), 5.23\left(\mathrm{t},{ }^{4} J_{\mathrm{H}, \mathrm{H}}=6.6 ; 1 \mathrm{H}\right.$, $\mathrm{HC}=\mathrm{C}), 6.94\left(\mathrm{~d},{ }^{2} J_{\mathrm{H}, \mathrm{H}}=13.5 ; 1 \mathrm{H}, \mathrm{N}=\mathrm{CH}_{2}\right), 7.36\left(\mathrm{~d},{ }^{2} J_{\mathrm{H}, \mathrm{H}}=13.5 ; 1 \mathrm{H}, \mathrm{N}=\mathrm{CH}_{2}\right), 7.64(\mathrm{~s}, 1 \mathrm{H}$, $\mathrm{HC}=\mathrm{N}) .{ }^{13} \mathrm{C}$ NMR $\delta 25.6\left(\mathrm{H}_{3} \underline{\mathrm{C}}-\mathrm{C}-\underline{\mathrm{C}} \mathrm{H}_{3}\right), 38.1\left(\mathrm{H}_{3} \mathrm{C}-\underline{\mathrm{C}}-\mathrm{CH}_{3}\right), 77.9\left(=\mathrm{CH}_{2}\right), 97.3(\mathrm{HC}=\mathrm{C}), 149.6$ $\left(\mathrm{N}=\mathrm{CH}_{2}\right), 169.4(\mathrm{HC}=\mathrm{N}), 207.2(=\mathrm{C}=)$.

(2,2,3-Trimethylpenta-3,4-dien-1-ylidene) formaldehyde hydrazone (5b). IR (film) $v_{\max } / \mathrm{cm}^{-1}$ 847, 991, 1109, 1196, 1383, 1456, $1606(\mathrm{C}=\mathrm{N}), 1637(\mathrm{C}=\mathrm{N}), 1952(=\mathrm{C}=), 2868,2927,2970 . \mathrm{MS}$ $\mathrm{m} / \mathrm{z}(\%) 151\left(\mathrm{M}^{+}+1 ; 4\right), 135$ (100), 122 (38), 107 (39), 79 (47), 67 (45), 53 (29), 41 (48). ${ }^{1} \mathrm{H}$ NMR $\delta 1.25\left(\mathrm{~s}, 6 \mathrm{H}, \mathrm{H}_{3} \mathrm{C}-\mathrm{C}-\mathrm{CH}_{3}\right), 1.67\left(\mathrm{t},{ }^{5} J_{\mathrm{H}, \mathrm{H}}=3.0 ; 3 \mathrm{H},=\mathrm{C}-\mathrm{CH}_{3}\right), 4.70\left(\mathrm{q},{ }^{5} J_{\mathrm{H}, \mathrm{H}}=3.0 ; 2 \mathrm{H}\right.$, $\left.=\mathrm{CH}_{2}\right), 6.93\left(\mathrm{~d},{ }^{2} J_{\mathrm{H}, \mathrm{H}}=13.5 ; 1 \mathrm{H}, \mathrm{N}=\mathrm{CH}_{2}\right), 7.35\left(\mathrm{~d},{ }^{2} J_{\mathrm{H}, \mathrm{H}}=13.5 ; 1 \mathrm{H}, \mathrm{N}=\mathrm{CH}_{2}\right), 7.57(\mathrm{~s}, 1 \mathrm{H}$, $\mathrm{HC}=\mathrm{N}) .{ }^{13} \mathrm{C}$ NMR $\delta 15.3\left(=\mathrm{C}-\underline{\mathrm{C}} \mathrm{H}_{3}\right), 24.2\left(\mathrm{H}_{3} \underline{\mathrm{C}}-\mathrm{C}-\underline{\mathrm{CH}}_{3}\right), 40.2\left(\mathrm{H}_{3} \mathrm{C}-\underline{\mathrm{C}}-\mathrm{CH}_{3}\right), 76.1\left(=\mathrm{CH}_{2}\right), 103.3$ $\left(=\underline{\mathrm{C}}-\mathrm{CH}_{3}\right), 149.3\left(\mathrm{~N}=\mathrm{CH}_{2}\right), 169.1(\mathrm{HC}=\mathrm{N}), 206.4(=\mathrm{C}=)$. 
(2,2-Dimethylpenta-3,4-dien-1-ylidene) acetaldehyde hydrazone (5c). IR (film) $v_{\max } / \mathrm{cm}^{-1}$ 847, 1147, 1309, 1377, 1433, 1464, $1651(\mathrm{C}=\mathrm{N}), 1954(=\mathrm{C}=), 2891,2929,2970 .{ }^{1} \mathrm{H}$ NMR $\delta 1.23$ $\left(\mathrm{s}, 6 \mathrm{H}, \mathrm{H}_{3} \mathrm{C}-\mathrm{C}-\mathrm{CH}_{3}\right), 2.01\left(\mathrm{~d},{ }^{3} J_{\mathrm{H}, \mathrm{H}}=5.3 ; 3 \mathrm{H}, \mathrm{HC}-\mathrm{CH}_{3}\right), 4.80\left(\mathrm{~d},{ }^{4} J_{\mathrm{H}, \mathrm{H}}=6.6 ; 2 \mathrm{H},=\mathrm{CH}_{2}\right), 5.22(\mathrm{t}$, $\left.{ }^{4} J_{\mathrm{H}, \mathrm{H}}=6.6 ; 1 \mathrm{H}, \mathrm{HC}=\mathrm{C}\right), 7.69(\mathrm{~s}, 1 \mathrm{H}, \mathrm{HC}=\mathrm{N}), 7.87\left(\mathrm{q},{ }^{3} J_{\mathrm{H}, \mathrm{H}}=5.5 ; 1 \mathrm{H}, \underline{\mathrm{HC}}-\mathrm{CH}_{3}\right) .{ }^{13} \mathrm{C} \mathrm{NMR} \delta 18.9$ $\left(\mathrm{HC}-\underline{\mathrm{CH}}{ }_{3}\right), 25.6\left(\mathrm{H}_{3} \underline{\mathrm{C}}-\mathrm{C}-\underline{\mathrm{C}} \mathrm{H}_{3}\right), 38.0\left(\mathrm{H}_{3} \mathrm{C}-\underline{\mathrm{C}}-\mathrm{CH}_{3}\right), 77.9\left(=\mathrm{CH}_{2}\right), 97.3(\mathrm{HC}=\mathrm{C}), 161.5\left(\mathrm{HC}_{-} \mathrm{CH}_{3}\right)$, $169.3(\mathrm{HC}=\mathrm{N}), 207.0(=\mathrm{C}=)$.

(2,2,3-Trimethylpenta-3,4-dien-1-ylidene) acetaldehyde hydrazone (5d). IR (film) $v_{\max } / \mathrm{cm}^{-1}$ 845, 1109, 1309, 1375, 1434, $1653(\mathrm{C}=\mathrm{N}), 1955(=\mathrm{C}=), 2929,2972 .{ }^{1} \mathrm{H}$ NMR $\delta 1.23(\mathrm{~s}, 6 \mathrm{H}$, $\left.\mathrm{H}_{3} \mathrm{C}-\mathrm{C}-\mathrm{CH}_{3}\right), 1.65\left(\mathrm{t},{ }^{5} J_{\mathrm{H}, \mathrm{H}}=3.1 ; 3 \mathrm{H},=\mathrm{C}-\mathrm{CH}_{3}\right), 1.95\left(\mathrm{~d},{ }^{3} J_{\mathrm{H}, \mathrm{H}}=5.3 ; 3 \mathrm{H}, \mathrm{HC}-\underline{\mathrm{H}}_{3}\right), 4.68\left(\mathrm{q},{ }^{5} J_{\mathrm{H}, \mathrm{H}}\right.$ $\left.=3.0 ; 2 \mathrm{H},=\mathrm{CH}_{2}\right), 7.62(\mathrm{~s}, 1 \mathrm{H}, \mathrm{HC}=\mathrm{N}), 7.85\left(\mathrm{q},{ }^{3} J_{\mathrm{H}, \mathrm{H}}=5.4 ; 1 \mathrm{H}, \underline{\mathrm{HC}}-\mathrm{CH}_{3}\right) .{ }^{13} \mathrm{C} \mathrm{NMR} \delta 15.3(=\mathrm{C}-$ $\left.\underline{\mathrm{CH}}_{3}\right), 18.9\left(\mathrm{HC}-\underline{\mathrm{CH}}{ }_{3}\right), 24.3\left(\mathrm{H}_{3} \underline{\mathrm{C}}-\mathrm{C}-\underline{\mathrm{CH}}_{3}\right), 40.1\left(\mathrm{H}_{3} \mathrm{C}-\underline{\mathrm{C}}-\mathrm{CH}_{3}\right), 76.0\left(=\mathrm{CH}_{2}\right), 103.4\left(=\underline{\mathrm{C}}-\mathrm{CH}_{3}\right)$, $160.9\left(\mathrm{HC}-\mathrm{CH}_{3}\right), 169.0(\mathrm{HC}=\mathrm{N}), 206.4(=\mathrm{C}=)$.

(2,2-Dimethylpenta-3,4-dien-1-ylidene) propionaldehyde hydrazone (5e). IR (film) $v_{\max } / \mathrm{cm}^{-}$ ${ }^{1} 847,901,1147,1317,1363,1462,1651(\mathrm{C}=\mathrm{N}), 1956(=\mathrm{C}=), 2885,2935,2970 . \mathrm{MS} \mathrm{m} / \mathrm{z}(\%)$ $165\left(\mathrm{M}^{+}+1\right.$; 84), 149 (91), 135 (62), 108 (100), 94 (88), 81 (66), 67 (51), 53 (38), 41 (64). ${ }^{1} \mathrm{H}$ NMR $\delta 1.11\left(\mathrm{t},{ }^{3} J_{\mathrm{H}, \mathrm{H}}=7.6 ; 3 \mathrm{H}, \mathrm{CH}_{2}-\mathrm{CH}_{3}\right), 1.22\left(\mathrm{~s}, 6 \mathrm{H}, \mathrm{H}_{3} \mathrm{C}-\mathrm{C}-\mathrm{CH}_{3}\right), 2.32\left(\mathrm{dq},{ }^{3} J_{\mathrm{H}, \mathrm{H}}=7.6 ;{ }^{3} J_{\mathrm{H}, \mathrm{H}}\right.$ $\left.=5.3 ; 2 \mathrm{H}, \underline{\mathrm{CH}}_{2}-\mathrm{CH}_{3}\right), 4.79\left(\mathrm{~d},{ }^{4} J_{\mathrm{H}, \mathrm{H}}=6.7 ; 2 \mathrm{H},=\mathrm{CH}_{2}\right), 5.21\left(\mathrm{t},{ }^{4} J_{\mathrm{H}, \mathrm{H}}=6.7 ; 1 \mathrm{H}, \mathrm{HC}=\mathrm{C}\right), 7.67(\mathrm{~s}$, $1 \mathrm{H}, \mathrm{HC}=\mathrm{N}), 7.80\left(\mathrm{t},{ }^{3} \mathrm{~J}_{\mathrm{H}, \mathrm{H}}=5.2 ; 1 \mathrm{H}, \underline{\mathrm{HC}}-\mathrm{CH}_{2}\right) .{ }^{13} \mathrm{C} \mathrm{NMR} \delta 10.5\left(\mathrm{CH}_{2}-\mathrm{CH}_{3}\right), 25.6\left(\mathrm{H}_{3} \underline{\mathrm{C}}-\mathrm{C}-\underline{\mathrm{C}} \mathrm{H}_{3}\right)$, $26.2\left(\underline{\mathrm{CH}}_{2}-\mathrm{CH}_{3}\right), 38.0\left(\mathrm{H}_{3} \mathrm{C}-\underline{\mathrm{C}}-\mathrm{CH}_{3}\right), 77.7\left(=\mathrm{CH}_{2}\right), 97.4(\mathrm{HC}=\mathrm{C}), 165.8\left(\mathrm{HC}-\mathrm{CH}_{2}\right), 168.9$ $(\mathrm{HC}=\mathrm{N}), 207.1(=\mathrm{C}=)$.

(2,2,3-Trimethylpenta-3,4-dien-1-ylidene) propionaldehyde hydrazone (5f). IR (film) $v_{\max } /$ $\mathrm{cm}^{-1} 847,1034,1109,1315,1373,1458,1649(\mathrm{C}=\mathrm{N}), 1954(=\mathrm{C}=), 2881,2937,2974 . \mathrm{MS} \mathrm{m} / \mathrm{z}$ (\%) $179\left(\mathrm{M}^{+}+1\right.$; 49), 163 (77), 149 (54), 122 (100), 108 (96), 97 (48), 81 (49), 67 (64), 55 (45), 41 (64). ${ }^{1} \mathrm{H}$ NMR $\delta 1.14\left(\mathrm{t},{ }^{3} J_{\mathrm{H}, \mathrm{H}}=7.6 ; 3 \mathrm{H}, \mathrm{CH}_{2}-\underline{\mathrm{H}}_{3}\right), 1.25\left(\mathrm{~s}, 6 \mathrm{H}, \mathrm{H}_{3} \mathrm{C}-\mathrm{C}-\mathrm{CH}_{3}\right), 1.67\left(\mathrm{t},{ }^{5} J_{\mathrm{H}, \mathrm{H}}=\right.$ $\left.3.0 ; 3 \mathrm{H},=\mathrm{C}-\mathrm{CH}_{3}\right), 2.35\left(\mathrm{dq},{ }^{3} J_{\mathrm{H}, \mathrm{H}}=7.5 ;{ }^{3} J_{\mathrm{H}, \mathrm{H}}=5.1 ; 2 \mathrm{H}, \underline{\mathrm{CH}}_{2}-\mathrm{CH}_{3}\right), 4.70\left(\mathrm{q},{ }^{5} J_{\mathrm{H}, \mathrm{H}}=3.0 ; 2 \mathrm{H}\right.$, $\left.=\mathrm{CH}_{2}\right), 7.63(\mathrm{~s}, 1 \mathrm{H}, \mathrm{HC}=\mathrm{N}), 7.81\left(\mathrm{t},{ }^{3} J_{\mathrm{H}, \mathrm{H}}=5.1 ; 1 \mathrm{H}, \underline{\mathrm{HC}}-\mathrm{CH}_{2}\right) .{ }^{13} \mathrm{C} \mathrm{NMR} \delta 10.5\left(\mathrm{CH}_{2}-\underline{C H}_{3}\right), 15.2$ $\left(=\mathrm{C}-\underline{\mathrm{CH}}_{3}\right), 24.3\left(\mathrm{H}_{3} \underline{\mathrm{C}}-\mathrm{C}-\underline{\mathrm{CH}}_{3}\right), 26.1\left(\underline{\mathrm{CH}}_{2}-\mathrm{CH}_{3}\right), 40.0\left(\mathrm{H}_{3} \mathrm{C}-\underline{\mathrm{C}}-\mathrm{CH}_{3}\right), 75.9\left(=\mathrm{CH}_{2}\right), 103.3(=\underline{\mathrm{C}}-$ $\left.\mathrm{CH}_{3}\right), 165.4\left(\mathrm{H} \underline{\mathrm{C}}-\mathrm{CH}_{2}\right), 168.6(\mathrm{HC}=\mathrm{N}), 206.3(=\mathrm{C}=)$.

(2,2-Dimethylpenta-3,4-dien-1-ylidene) isobutyraldehyde hydrazone (5g). IR (film) $v_{\max } /$ $\mathrm{cm}^{-1} 847,1034,1363,1464,1651(\mathrm{C}=\mathrm{N}), 1955(=\mathrm{C}=), 2872,2931,2966 . \mathrm{MS} \mathrm{m} / \mathrm{z}(\%) 179$ $\left(\mathrm{M}^{+}+1 ; 75\right), 163$ (55), 135 (45), 108 (100), 94 (69), 79 (44), 67 (29), 53 (34), 41 (51). ${ }^{1} \mathrm{H}$ NMR $\delta$ $1.06\left(\mathrm{~d},{ }^{3} \mathrm{~J}_{\mathrm{H}, \mathrm{H}}=6.9 ; 6 \mathrm{H}, \underline{\mathrm{H}}_{3} \mathrm{C}-\mathrm{CH}-\underline{\mathrm{H}}_{3}\right), 1.18\left(\mathrm{~s}, 6 \mathrm{H}, \mathrm{H}_{3} \mathrm{C}-\mathrm{C}-\mathrm{CH}_{3}\right), 2.40-2.55\left(\mathrm{~m}, 1 \mathrm{H}, \mathrm{H}_{3} \mathrm{C}-\mathrm{C} \underline{\mathrm{H}}-\right.$ $\left.\mathrm{CH}_{3}\right), 4.74\left(\mathrm{~d},{ }^{4} J_{\mathrm{H}, \mathrm{H}}=6.6 ; 2 \mathrm{H},=\mathrm{CH}_{2}\right), 5.17\left(\mathrm{t},{ }^{4} J_{\mathrm{H}, \mathrm{H}}=6.6 ; 1 \mathrm{H}, \mathrm{HC}=\mathrm{C}\right), 7.61(\mathrm{~s}, 1 \mathrm{H}, \mathrm{HC}=\mathrm{N}), 7.63$ $\left(\mathrm{d},{ }^{3} J_{\mathrm{H}, \mathrm{H}}=5.9 ; 1 \mathrm{H},=\mathrm{C} \underline{\mathrm{H}}-\mathrm{CH}\right) .{ }^{13} \mathrm{C}$ NMR $\delta 19.7\left(\mathrm{H}_{3} \underline{\mathrm{C}}-\mathrm{CH}-\underline{\mathrm{CH}} 3\right), 25.6\left(\mathrm{H}_{3} \underline{\mathrm{C}}-\mathrm{C}-\underline{C H}_{3}\right), 31.7\left(\mathrm{H}_{3} \mathrm{C}-\right.$ $\left.\underline{\mathrm{CH}}-\mathrm{CH}_{3}\right), 38.0\left(\mathrm{H}_{3} \mathrm{C}-\underline{\mathrm{C}}-\mathrm{CH}_{3}\right), 77.7\left(=\mathrm{CH}_{2}\right), 97.4(\mathrm{HC}=\mathrm{C}), 168.8(=\underline{\mathrm{CH}}-\mathrm{CH}), 169.2(\mathrm{HC}=\mathrm{N})$, $207.1(=\mathrm{C}=)$.

(2,2,3-Trimethylpenta-3,4-dien-1-ylidene) isobutyraldehyde hydrazone (5h). IR (film) $v_{\max } /$ $\mathrm{cm}^{-1} 847,1109,1363,1466,1647(\mathrm{C}=\mathrm{N}), 1954(=\mathrm{C}=), 2872,2931,2968 .{ }^{1} \mathrm{H}$ NMR $\delta 1.06(\mathrm{~d}$, $\left.{ }^{3} J_{\mathrm{H}, \mathrm{H}}=6.6 ; 6 \mathrm{H}, \underline{\mathrm{H}}_{3} \mathrm{C}-\mathrm{CH}-\underline{\mathrm{H}}_{3}\right), 1.18\left(\mathrm{~s}, 6 \mathrm{H}, \mathrm{H}_{3} \mathrm{C}-\mathrm{C}-\mathrm{CH}_{3}\right), 1.61\left(\mathrm{t},{ }^{5} J_{\mathrm{H}, \mathrm{H}}=3.0 ; 3 \mathrm{H},=\mathrm{C}_{-} \mathrm{CH}_{3}\right), 2.40$ $-2.55\left(\mathrm{~m}, 1 \mathrm{H}, \mathrm{H}_{3} \mathrm{C}-\mathrm{CH}-\mathrm{CH}_{3}\right), 4.63\left(\mathrm{q},{ }^{5} J_{\mathrm{H}, \mathrm{H}}=2.9 ; 2 \mathrm{H},=\mathrm{CH}_{2}\right), 7.54(\mathrm{~s}, 1 \mathrm{H}, \mathrm{HC}=\mathrm{N}), 7.62\left(\mathrm{~d},{ }^{3} J_{\mathrm{H}, \mathrm{H}}\right.$ 
$=5.3 ; 1 \mathrm{H},=\mathrm{C} \underline{\mathrm{H}}-\mathrm{CH}) .{ }^{13} \mathrm{C}$ NMR $\delta 15.3\left(=\mathrm{C}-\underline{\mathrm{C}} \mathrm{H}_{3}\right), 19.7\left(\mathrm{H}_{3} \underline{\mathrm{C}}-\mathrm{CH}-\underline{\mathrm{C}} \mathrm{H}_{3}\right), 24.3\left(\mathrm{H}_{3} \underline{\mathrm{C}}-\mathrm{C}-\underline{\mathrm{C}} \mathrm{H}_{3}\right), 31.7$ $\left(\mathrm{H}_{3} \mathrm{C}-\underline{\mathrm{CH}}-\mathrm{CH}_{3}\right), 40.1 \quad\left(\mathrm{H}_{3} \mathrm{C}-\underline{\mathrm{C}}-\mathrm{CH}_{3}\right), 75.9\left(=\mathrm{CH}_{2}\right), 103.4\left(=\underline{\mathrm{C}}-\mathrm{CH}_{3}\right), 168.4(=\underline{\mathrm{C}} \mathrm{H}-\mathrm{CH}), 168.8$ $(\mathrm{HC}=\mathrm{N}), 206.4(=\mathrm{C}=)$.

(2,2-Dimethylpenta-3,4-dien-1-ylidene) crotonaldehyde hydrazone (5i). IR (film) $v_{\max } / \mathrm{cm}^{-1}$ 847, 958, 987, 1172, 1298, 1363, 1385, 1444, 1458, $1626(\mathrm{C}=\mathrm{N}), 1655(\mathrm{C}=\mathrm{N}), 1954(=\mathrm{C}=)$, 2870, 2929, 2970, 3033. MS m/z (\%) 177 (M+1; 75), 161 (100), 145 (17), 132 (19), 108 (37), 94 (42), 79 (22), 67 (27), 53 (28), 41 (43). ${ }^{1} \mathrm{H}$ NMR $\delta 1.22$ (s, 6H, $\left.\mathrm{H}_{3} \mathrm{C}-\mathrm{C}-\mathrm{CH}_{3}\right), 1.87$ (d, ${ }^{3} J_{\mathrm{H}, \mathrm{H}}=$ $\left.4.6 ; 3 \mathrm{H},=\mathrm{CH}-\mathrm{CH}_{3}\right), 4.78\left(\mathrm{~d},{ }^{4} J_{\mathrm{H}, \mathrm{H}}=6.6 ; 2 \mathrm{H},=\mathrm{CH}_{2}\right), 5.21\left(\mathrm{t},{ }^{4} J_{\mathrm{H}, \mathrm{H}}=6.6 ; 1 \mathrm{H}, \mathrm{HC}=\mathrm{C}\right), 6.11-6.36$ $(\mathrm{m}, 2 \mathrm{H}, \mathrm{CH}=\mathrm{CH}), 7.71(\mathrm{~s}, 1 \mathrm{H}, \mathrm{HC}=\mathrm{N}), 8.03\left(\mathrm{~d},{ }^{3} J_{\mathrm{H}, \mathrm{H}}=8.6 ; 1 \mathrm{H}, \mathrm{N}=\mathrm{C} \underline{H}-\mathrm{CH}\right) .{ }^{13} \mathrm{C}$ NMR $\delta 18.6$ $\left(=\mathrm{CH}-\underline{\mathrm{CH}}_{3}\right), 25.4\left(\underline{\mathrm{CH}}_{3}-\mathrm{C}-\underline{\mathrm{C}} \mathrm{H}_{3}\right), 37.8\left(\mathrm{CH}_{3}-\underline{\mathrm{C}}-\mathrm{CH}_{3}\right), 77.5\left(=\mathrm{CH}_{2}\right), 97.2(\mathrm{H} \underline{\mathrm{C}}=\mathrm{C}), 128.9(=\underline{\mathrm{C}} \mathrm{H}-$ $\left.\mathrm{CH}_{3}\right), 141.9(\mathrm{~N}=\mathrm{CH}-\underline{\mathrm{CH}}), 162.7(\mathrm{~N}=\underline{\mathrm{CH}}-\mathrm{CH}), 169.4(\mathrm{HC}=\mathrm{N}), 206.8(=\mathrm{C}=)$.

(2,2,3-Trimethylpenta-3,4-dien-1-ylidene) crotonaldehyde hydrazone (5j). IR (film) $v_{\max } /$ $\mathrm{cm}^{-1} 847,960,986,1109,1373,1446,1626(\mathrm{C}=\mathrm{N}), 1653(\mathrm{C}=\mathrm{N}), 1952(=\mathrm{C}=), 2872,2929,2972$, 3034. MS m/z (\%) $191\left(\mathrm{M}^{+}+1\right.$; 52), 175 (100), 159 (18), 146 (19), 122 (29), 108 (43), 79 (17), 67 (34), $53(24), 41(45) .{ }^{1} \mathrm{H}$ NMR $\delta 1.23\left(\mathrm{~s}, 6 \mathrm{H}, \mathrm{H}_{3} \mathrm{C}-\mathrm{C}-\mathrm{CH}_{3}\right), 1.64\left(\mathrm{t},{ }^{5} J_{\mathrm{H}, \mathrm{H}}=3.1 ; 3 \mathrm{H},=\mathrm{C}_{-} \mathrm{CH}_{3}\right)$, $1.88\left(\mathrm{~d},{ }^{3} J_{\mathrm{H}, \mathrm{H}}=5.0 ; 3 \mathrm{H},=\mathrm{CH}-\underline{\mathrm{C}}_{3}\right), 4.67\left(\mathrm{q},{ }^{5} J_{\mathrm{H}, \mathrm{H}}=2.9 ; 2 \mathrm{H},=\mathrm{CH}_{2}\right), 6.10-6.34(\mathrm{~m}, 2 \mathrm{H}$, $\mathrm{CH}=\mathrm{CH}), 7.66(\mathrm{~s}, 1 \mathrm{H}, \mathrm{HC}=\mathrm{N}), 8.04\left(\mathrm{~d},{ }^{3} J_{\mathrm{H}, \mathrm{H}}=8.6 ; 1 \mathrm{H}, \mathrm{N}=\mathrm{C} \underline{\mathrm{H}}-\mathrm{CH}\right) .{ }^{13} \mathrm{C} \mathrm{NMR} \delta 15.3\left(=\mathrm{C}-\underline{\mathrm{CH}}_{3}\right)$, $18.8\left(=\mathrm{CH}-\underline{\mathrm{CH}}_{3}\right), 24.3\left(\underline{\mathrm{CH}}_{3}-\mathrm{C}-\underline{\mathrm{C}} \mathrm{H}_{3}\right), 40.2\left(\mathrm{CH}_{3}-\underline{\mathrm{C}}-\mathrm{CH}_{3}\right), 76.0\left(=\mathrm{CH}_{2}\right), 103.4\left(=\underline{\mathrm{C}}-\mathrm{CH}_{3}\right), 129.2$ $\left(=\underline{\mathrm{CH}}-\mathrm{CH}_{3}\right), 142.1(\mathrm{~N}=\mathrm{CH}-\underline{\mathrm{CH}}), 162.7(\mathrm{~N}=\underline{\mathrm{CH}}-\mathrm{CH}), 169.5(\mathrm{HC}=\mathrm{N}), 206.4(=\mathrm{C}=)$.

(2,2-Dimethylpenta-3,4-dien-1-ylidene) cyclopentanone hydrazone (5k). IR (film) $v_{\max } / \mathrm{cm}^{-1}$ 847, 1201, 1362, 1383, 1423, 1454, 1466, $1659(\mathrm{C}=\mathrm{N}), 1954(=\mathrm{C}=), 2870,2885,2937,2964$. MS m/z (\%) $191\left(\mathrm{M}^{+}+1 ; 100\right), 175$ (86), 120 (12), 108 (75), 94 (39), 82 (25), 67 (30), 55 (29), 41 (22). ${ }^{1} \mathrm{H}$ NMR $\delta 1.13\left(\mathrm{~s}, 6 \mathrm{H}, \mathrm{H}_{3} \mathrm{C}-\mathrm{C}-\mathrm{CH}_{3}\right), 1.60-1.70\left(\mathrm{~m}, 4 \mathrm{H}, 2 \times \mathrm{CH}_{2}\right), 2.25-2.40(\mathrm{~m}, 4 \mathrm{H}$, $\left.2 \times \mathrm{CH}_{2}\right), 4.68\left(\mathrm{~d},{ }^{4} J_{\mathrm{H}, \mathrm{H}}=6.6 ; 2 \mathrm{H},=\mathrm{CH}_{2}\right), 5.12\left(\mathrm{t},{ }^{4} J_{\mathrm{H}, \mathrm{H}}=6.6 ; 1 \mathrm{H}, \mathrm{HC}=\mathrm{C}\right), 7.38(\mathrm{~s}, 1 \mathrm{H}, \mathrm{HC}=\mathrm{N})$. ${ }^{13} \mathrm{C}$ NMR $\delta 24.4\left(\mathrm{CH}_{2}\right), 24.6\left(\mathrm{CH}_{2}\right), 25.5\left(\mathrm{H}_{3} \underline{\mathrm{C}}-\mathrm{C}-\underline{\mathrm{C}} \mathrm{H}_{3}\right), 29.3\left(\mathrm{CH}_{2}\right), 33.2\left(\mathrm{CH}_{2}\right), 37.9\left(\mathrm{H}_{3} \mathrm{C}-\underline{\mathrm{C}}-\right.$ $\left.\mathrm{CH}_{3}\right), 77.3\left(=\mathrm{CH}_{2}\right), 97.4(\mathrm{H} \underline{\mathrm{C}}=\mathrm{C}), 164.9(\mathrm{HC}=\mathrm{N}), 178.5(\mathrm{~N}=\mathrm{C}), 206.8(=\mathrm{C}=)$.

(2,2,3-Trimethylpenta-3,4-dien-1-ylidene) cyclopentanone hydrazone (5I). IR (film) $v_{\max } /$ $\mathrm{cm}^{-1} 847,1109,1201,1371,1425,1452,1657(\mathrm{C}=\mathrm{N}), 1954(=\mathrm{C}=), 2872,2937,2966 . \mathrm{MS} \mathrm{m} / \mathrm{z}$ (\%) $205\left(\mathrm{M}^{+}+1 ; 100\right), 189$ (95), 122 (44), 108 (48), 82 (20), 67 (25), 55 (25), 41 (19). ${ }^{1} \mathrm{H}$ NMR $\delta$ $1.24\left(\mathrm{~s}, 6 \mathrm{H}, \mathrm{H}_{3} \mathrm{C}-\mathrm{C}-\mathrm{CH}_{3}\right), 1.68\left(\mathrm{t},{ }^{5} \mathrm{~J}_{\mathrm{H}, \mathrm{H}}=3.0 ; 3 \mathrm{H},=\mathrm{C}^{-\mathrm{CH}_{3}}\right), 1.71-1.84\left(\mathrm{~m}, 4 \mathrm{H}, 2 \times \mathrm{CH}_{2}\right), 2.37-$ $2.52\left(\mathrm{~m}, 4 \mathrm{H}, 2 \times \mathrm{CH}_{2}\right), 4.69\left(\mathrm{q},{ }^{5} J_{\mathrm{H}, \mathrm{H}}=3.1 ; 2 \mathrm{H},=\mathrm{CH}_{2}\right), 7.42(\mathrm{~s}, 1 \mathrm{H}, \mathrm{HC}=\mathrm{N}) .{ }^{13} \mathrm{C}$ NMR $\delta 15.3$ $\left(=\mathrm{C}-\underline{\mathrm{CH}_{3}}\right), 24.4\left(\mathrm{H}_{3} \underline{\mathrm{C}}-\mathrm{C}-\underline{\mathrm{C}} \mathrm{H}_{3}\right), 24.7\left(\mathrm{CH}_{2}\right), 24.8\left(\mathrm{CH}_{2}\right), 29.7\left(\mathrm{CH}_{2}\right), 33.5\left(\mathrm{CH}_{2}\right), 40.3\left(\mathrm{H}_{3} \mathrm{C}-\underline{\mathrm{C}}-\right.$ $\left.\mathrm{CH}_{3}\right), 75.8\left(=\mathrm{CH}_{2}\right), 103.5\left(=\underline{\mathrm{C}}-\mathrm{CH}_{3}\right), 164.8(\mathrm{HC}=\mathrm{N}), 178.4(\mathrm{~N}=\mathrm{C}), 206.3(=\mathrm{C}=)$.

(2,2-Dimethylpenta-3,4-dien-1-ylidene) cyclohexanone hydrazone (5m). IR (film) $v_{\max } / \mathrm{cm}^{-1}$ 845, 951, 1134, 1244, 1317, 1362, 1383, 1448, $1643(\mathrm{C}=\mathrm{N}), 1954(=\mathrm{C}=), 2860,2889,2933$, 2968. MS m/z (\%) 205 ( $\left.\mathrm{M}^{+}+1 ; 100\right), 189$ (92), 162 (13), 147 (19), 136 (24), 122 (19), 108 (73), 96 (67), 79 (40), 69 (33), 55 (45), 41 (44). ${ }^{1} \mathrm{H}$ NMR $\delta 1.25$ (s, 6H, $\left.\mathrm{CH}_{3}-\mathrm{C}-\mathrm{CH}_{3}\right), 1.48-1.87$ (m, $\left.6 \mathrm{H}, 3 \times \mathrm{CH}_{2}\right), 2.21-2.43\left(\mathrm{~m}, 2 \mathrm{H}, \mathrm{CH}_{2}\right), 2.46-2.71\left(\mathrm{~m}, 2 \mathrm{H}, \mathrm{CH}_{2}\right), 4.80\left(\mathrm{~d},{ }^{4} J_{\mathrm{H}, \mathrm{H}}=6.6 ; 2 \mathrm{H}\right.$, $\left.=\mathrm{CH}_{2}\right), 5.25\left(\mathrm{t},{ }^{4} J_{\mathrm{H}, \mathrm{H}}=6.5 ; 1 \mathrm{H}, \mathrm{HC}=\mathrm{C}\right), 7.50(\mathrm{~s}, 1 \mathrm{H}, \mathrm{HC}=\mathrm{N}) .{ }^{13} \mathrm{C} \mathrm{NMR} \delta 25.7\left(\mathrm{H}_{3} \underline{\mathrm{C}}-\mathrm{C}-\underline{\mathrm{C}} \mathrm{H}_{3}\right)$, 
$26.1\left(\mathrm{CH}_{2}\right), 26.5\left(\mathrm{CH}_{2}\right), 27.5\left(\mathrm{CH}_{2}\right), 28.5\left(\mathrm{CH}_{2}\right) 36.0\left(\mathrm{CH}_{2}\right), 38.4\left(\mathrm{H}_{3} \mathrm{C}-\underline{\mathrm{C}}-\mathrm{CH}_{3}\right), 77.6\left(=\mathrm{CH}_{2}\right)$, $97.7(\mathrm{HC}=\mathrm{C}), 165.6(\mathrm{HC}=\mathrm{N}), 171.4(\mathrm{~N}=\mathrm{C}), 207.1(=\mathrm{C}=)$.

(2,2,3-Trimethylpenta-3,4-dien-1-ylidene) cyclohexanone hydrazone (5n). IR (film) $v_{\max } /$ $\mathrm{cm}^{-1} 847,1109,1317,1371,1448,1643(\mathrm{C}=\mathrm{N}), 1954(=\mathrm{C}=), 2860,2889,2929,2970 . \mathrm{MS} \mathrm{m} / \mathrm{z}$ (\%) $219\left(\mathrm{M}^{+}+1\right.$; 100), 203 (79), 150 (12), 136 (13), 122 (65), 108 (35), 96 (56), 79 (19), 67 (22), 55 (29), 41 (22). ${ }^{1} \mathrm{H}$ NMR $\delta 1.21\left(\mathrm{~s}, 6 \mathrm{H}, \mathrm{CH}_{3}-\mathrm{C}-\mathrm{CH}_{3}\right), 1.54-1.63\left(\mathrm{~m}, 4 \mathrm{H}, 2 \times \mathrm{CH}_{2}\right), 1.63\left(\mathrm{t},{ }^{5} J_{\mathrm{H}, \mathrm{H}}\right.$ $\left.=3.0 ; 3 \mathrm{H},=\mathrm{C}-\mathrm{CH}_{3}\right), 1.65-1.78\left(\mathrm{~m}, 2 \mathrm{H}, \mathrm{CH}_{2}\right), 2.22-2.34\left(\mathrm{~m}, 2 \mathrm{H}, \mathrm{CH}_{2}\right), 2.47-2.57(\mathrm{~m}, 2 \mathrm{H}$, $\left.\mathrm{CH}_{2}\right), 4.64\left(\mathrm{q},{ }^{5} J_{\mathrm{H}, \mathrm{H}}=3.0 ; 2 \mathrm{H},=\mathrm{CH}_{2}\right), 7.38(\mathrm{~s}, 1 \mathrm{H}, \mathrm{HC}=\mathrm{N}),{ }^{13} \mathrm{C}$ NMR $\delta 15.3\left(=\mathrm{C}-\underline{C H}_{3}\right), 24.3$ $\left(\mathrm{H}_{3} \underline{\mathrm{C}}-\mathrm{C}-\mathrm{CH}_{3}\right), 26.0\left(\mathrm{CH}_{2}\right), 26.5\left(\mathrm{CH}_{2}\right), 27.5\left(\mathrm{CH}_{2}\right), 28.5\left(\mathrm{CH}_{2}\right), 35.9\left(\mathrm{CH}_{2}\right), 40.4\left(\mathrm{H}_{3} \mathrm{C}-\underline{\mathrm{C}}-\mathrm{CH}_{3}\right)$, $75.8\left(=\mathrm{CH}_{2}\right), 103.6\left(=\underline{\mathrm{C}}-\mathrm{CH}_{3}\right), 165.0(\mathrm{HC}=\mathrm{N}), 170.8(\mathrm{~N}=\mathrm{C}), 206.3(=\mathrm{C}=)$.

\section{General procedure for the preparation of 7 a-c}

A non-symmetrical azine 5 (1 mmol) in dry xylene $(20 \mathrm{ml})$ was heated under reflux and after removal of solvent under vacuum the liquid residue was separated by liquid chromatography on silica gel. In all cases there were isolated brownish liquids.

4,5,5-Trimethyl-5,6-dihydro-4H-pyrrolo[1,2-b]pyrazole (7a). IR (film) $v_{\max } / \mathrm{cm}^{-1}$ 940, 1171, 1341, 1372, 1389, 1464, 1485, 1536, 2873, 2936, 2965. GC-MS m/z (\%) $150\left(\mathrm{M}^{+} ; 14\right), 135$ (14), 109 (11), 95 (100), 83 (19), 65 (12), 41 (18). ${ }^{1} \mathrm{H}$ NMR $\delta 1.05\left(\mathrm{CH}_{3}\right), 1.16\left(\mathrm{~d},{ }^{3} J_{\mathrm{H}, \mathrm{H}}=7.3 ; 3 \mathrm{H}, \mathrm{HC}-\right.$ $\left.\underline{\mathrm{CH}}_{3}\right), 1.23\left(\mathrm{CH}_{3}\right), 2.86\left(\mathrm{q},{ }^{3} J_{\mathrm{H}, \mathrm{H}}=7.2 ; 1 \mathrm{H}, \underline{\mathrm{HC}}-\mathrm{CH}_{3}\right), 3.80-3.90\left(\mathrm{~m}, \mathrm{CH}_{2}\right), 5.92$ (broad s, $\mathrm{C}=\mathrm{CH}), 7.46\left(\right.$ broad s, N=CH). ${ }^{13} \mathrm{C}$ NMR $\delta 13.1\left(\mathrm{HC}_{-} \underline{\mathrm{CH}}_{3}\right), 22.7\left(\mathrm{CH}_{3}\right), 26.9\left(\mathrm{CH}_{3}\right), 41.9(\mathrm{HC}-$ $\left.\mathrm{CH}_{3}\right), 46.4\left(-\underline{\mathrm{C}}-\left(\mathrm{CH}_{3}\right)_{2}\right), 60.9\left(\mathrm{CH}_{2}\right), 98.4(-\mathrm{C}=\underline{\mathrm{CH}}), 142.7(\mathrm{~N}=\mathrm{CH}), 150.0(\mathrm{~N}-\mathrm{C}=)$.

2,4,5,5-Tetramethyl-5,6-dihydro-4H-pyrrolo[1,2-b]pyrazole (7b). IR (film) $v_{\max } / \mathrm{cm}^{-1} 1009$, 1163, 1313, 1369, 1444, 1543, 2872, 2927, 2960. GC-MS m/z (\%) $164\left(\mathrm{M}^{+} ; 27\right), 149$ (27), 123 (25), 109 (100), 79 (15), 41 (12). ${ }^{1} \mathrm{H}$ NMR $\delta 0.96\left(\mathrm{CH}_{3}\right), 1.05\left(\mathrm{~d},{ }^{3} J_{\mathrm{H}, \mathrm{H}}=7.3 ; 3 \mathrm{H}, \mathrm{HC}-\underline{\mathrm{C}}_{3}\right), 1.12$ $\left(\mathrm{CH}_{3}\right), 2.17\left(=\mathrm{C}-\mathrm{CH}_{3}\right), 2.72\left(\mathrm{q},{ }^{3} \mathrm{~J}_{\mathrm{H}, \mathrm{H}}=7.2 ; 1 \mathrm{H}, \underline{\mathrm{HC}}-\mathrm{CH}_{3}\right), 3.65-3.75\left(\mathrm{~m}, \mathrm{CH}_{2}\right), 5.62($ broad s, $=\mathrm{CH}) .{ }^{13} \mathrm{C}$ NMR $\delta 12.8\left(\mathrm{HC}-\underline{C H}_{3}\right), 14.1\left(=\mathrm{C}-\underline{\mathrm{C}} \mathrm{H}_{3}\right), 22.4\left(\mathrm{CH}_{3}\right), 26.6\left(\mathrm{CH}_{3}\right), 41.7\left(\mathrm{HC}-\mathrm{CH}_{3}\right), 45.5$ $\left(-\underline{\mathrm{C}}-\left(\mathrm{CH}_{3}\right)_{2}\right), 60.4\left(\mathrm{CH}_{2}\right), 97.6(=\mathrm{CH}), 150.6(\mathrm{~N}-\mathrm{C}), 151.8(\mathrm{~N}=\mathrm{C})$.

2-Isopropyl-4,5,5-trimethyl-5,6-dihydro-4H-pyrrolo[1,2-b]pyrazole (7c). IR (film) $v_{\max } / \mathrm{cm}^{-1}$ 924, 1082, 1173, 1294, 1377, 1462, 1541, 2870, 2926, 2966. GC-MS m/z (\%) $192\left(\mathrm{M}^{+} ; 48\right), 177$ (100), 164 (12), 149 (12), 137 (43), 121 (30), 95 (16), 41 (11). ${ }^{1} \mathrm{H}$ NMR $\delta 1.03\left(\mathrm{CH}_{3}\right), 1.13$ (d, $\left.{ }^{3} J_{\mathrm{H}, \mathrm{H}}=7.3 ; 3 \mathrm{H}, \mathrm{HC}-\underline{\mathrm{H}}_{3}\right), 1.20\left(\mathrm{CH}_{3}\right), 1.24\left(\mathrm{~d},{ }^{3} J_{\mathrm{H}, \mathrm{H}}=6.9 ; 6 \mathrm{H}, \mathrm{HC}-\left(\mathrm{CH}_{3}\right)_{2}\right), 2.81\left(\mathrm{q},{ }^{3} J_{\mathrm{H}, \mathrm{H}}=7.2\right.$; $\left.1 \mathrm{H}, \underline{\mathrm{HC}}-\mathrm{CH}_{3}\right), 2.85-3.05\left(\mathrm{~m}, \underline{\mathrm{HC}}-\left(\mathrm{CH}_{3}\right)_{2}\right), 3.75-3.85\left(\mathrm{~m}, \mathrm{CH}_{2}\right), 5.73$ (broad s, $\left.=\mathrm{CH}\right) .{ }^{13} \mathrm{C}$ NMR $\delta 13.0\left(\mathrm{HC}^{-} \mathrm{CH}_{3}\right), 22.8\left(\mathrm{CH}_{3}\right), 23.1\left(\mathrm{CH}_{3}\right), 23.2\left(\mathrm{CH}_{3}\right), 26.9\left(\mathrm{CH}_{3}\right), 28.6\left(\mathrm{HC}-\left(\mathrm{CH}_{3}\right)_{2}\right), 42.1(\mathrm{H} \underline{\mathrm{C}}-$ $\left.\mathrm{CH}_{3}\right), 45.8\left(-\underline{\mathrm{C}}-\left(\mathrm{CH}_{3}\right)_{2}\right), 60.8\left(\mathrm{CH}_{2}\right), 94.9(=\mathrm{CH}), 150.4(\mathrm{~N}-\mathrm{C}), 163.2(\mathrm{~N}=\mathrm{C})$.

\section{General procedure for the preparation of 8a-i and 9}

A mixture of a non-symmetrical azine $\mathbf{5}$ and dipolarophile in dry xylene was heated to reflux. At the end of the reaction solvent was removed under vacuum. The residue was separated by liquid chromatography on silica gel $(\mathbf{8 a}, \mathbf{c}-\mathbf{e}, \mathbf{g}, \mathbf{9})$ or recrystallized $(\mathbf{8 b}, \mathbf{f}, \mathbf{h}, \mathbf{i})$ to give white microcrystals 
but in some cases $(\mathbf{8 e}, \mathbf{g}, \mathbf{9})$ there wasn't successful crystallization of these products. More data are provided in Table 4.

5,5-Dimethyl-3-phenyl-1,3,10-triazatricyclo[5.2.1 $\left.{ }^{\mathbf{4 , 7}} \cdot 0^{\mathbf{1 , 1 0}}\right]$ dec-6-en-2-one (8a). MP 139 - 142 ${ }^{\circ} \mathrm{C} . \mathrm{IR}\left(\mathrm{CHCl}_{3}\right) v_{\max } / \mathrm{cm}^{-1} 1173,1311,1392,1456,1500,1597,1707(\mathrm{C}=\mathrm{O}), 2873,2908,2937$, 2964. MS m/z (\%) 255 (M+; 85), 240 (100), 185 (24), 135 (28), 119 (16), 108 (32), 104 (47), 77 (65). HRMS calcd. for $\mathrm{C}_{15} \mathrm{H}_{18} \mathrm{~N}_{3} \mathrm{O}^{+}$256.1450; found 256.1447. ${ }^{1} \mathrm{H}$ NMR $\delta 0.82\left(\mathrm{~s}, 3 \mathrm{H}, \mathrm{CH}_{3}\right)$, $1.38\left(\mathrm{~s}, 3 \mathrm{H}, \mathrm{CH}_{3}\right), 2.50-2.68\left(\mathrm{~m}, 2 \mathrm{H},=\mathrm{C}-\mathrm{CH}_{2}\right), 3.61-3.76\left(\mathrm{~m}, 1 \mathrm{H}, \mathrm{N}-\mathrm{CH}_{2}\right), 4.16-4.28(\mathrm{~m}$, $\left.1 \mathrm{H}, \mathrm{N}-\mathrm{CH}_{2}\right), 4.72$ (broad s, 1H, =CH), $5.41(\mathrm{~s}, \mathrm{~N}-\mathrm{CH}-\mathrm{N}), 7.10-7.24(\mathrm{~m}, 1 \mathrm{H}, \mathrm{Ph}), 7.29-7.47$ $(\mathrm{m}, 4 \mathrm{H}, \mathrm{Ph}) .{ }^{13} \mathrm{C}$ NMR $\delta 23.8\left(=\mathrm{C}-\underline{\mathrm{CH}}_{2}\right), 24.3\left(\mathrm{CH}_{3}\right), 27.3\left(\mathrm{CH}_{3}\right), 49.6\left(\mathrm{~N}-\mathrm{CH}_{2}\right), 55.4\left(\underline{\mathrm{C}}-\left(\mathrm{CH}_{3}\right)_{2}\right)$, $84.8(\mathrm{~N}-\mathrm{CH}-\mathrm{N}), 110.4(=\mathrm{CH}), 122.6(\mathrm{~s}, 2 \times \mathrm{CH}, \mathrm{Ph}), 125.5(\mathrm{~s}, \mathrm{CH}, \mathrm{Ph}), 129.1$ (s, 2×CH, Ph), $138.8(\mathrm{C}, \mathrm{Ph}), 149.3\left(=\underline{\mathrm{C}}-\mathrm{CH}_{2}\right), 162.5(\mathrm{C}=\mathrm{O})$.

5,5,6-Trimethyl-3-phenyl-1,3,10-triazatricyclo[5.2.1 $\left.{ }^{4,7} \cdot 0^{1,10}\right]$ dec-6-en-2-one (8b). MP 144 $145^{\circ} \mathrm{C}$. IR $\left(\mathrm{CHCl}_{3}\right) v_{\max } / \mathrm{cm}^{-1} 1146,1294,1323,1383,1439,1466,1502,1599,1709(\mathrm{C}=\mathrm{O})$, 2860, 2935, 2966. MS m/z (\%) 269 (M+; 49), 254 (56), 162 (32), 150 (53), 135 (28), 122 (29), 104 (28), 91 (31), 77 (100), 67 (22), 51 (64), 41 (93). HRMS calcd. for $\mathrm{C}_{16} \mathrm{H}_{20} \mathrm{~N}_{3} \mathrm{O}^{+} 270.1606$; found 270.1590. ${ }^{1} \mathrm{H}$ NMR $\delta 0.73\left(\mathrm{~s}, 3 \mathrm{H}, \underline{\mathrm{H}}_{3} \mathrm{C}-\mathrm{C}-\mathrm{CH}_{3}\right), 1.29\left(\mathrm{~s}, 3 \mathrm{H}, \mathrm{H}_{3} \mathrm{C}-\mathrm{C}-\mathrm{CH}_{3}\right), 1.50\left(\mathrm{dd},{ }^{5} J_{\mathrm{H}, \mathrm{H}}\right.$ $\left.=2.0 ;{ }^{5} J_{\mathrm{H}, \mathrm{H}}=0.7 ; 3 \mathrm{H}, \mathrm{H}_{3} \mathrm{C}-\mathrm{C}=\right), 2.37-2.65\left(\mathrm{~m}, 2 \mathrm{H},=\mathrm{C}-\mathrm{CH}_{2}\right), 3.64\left(\mathrm{ddd},{ }^{2} J_{\mathrm{H}, \mathrm{H}}=11.9 ;{ }^{3} J_{\mathrm{H}, \mathrm{H}}=\right.$ $\left.5.4 ;{ }^{3} J_{\mathrm{H}, \mathrm{H}}=1.9 ; 1 \mathrm{H}, \mathrm{N}-\mathrm{CH}_{2}\right), 4.12\left(\mathrm{ddd},{ }^{2} J_{\mathrm{H}, \mathrm{H}}=9.1 ;{ }^{3} J_{\mathrm{H}, \mathrm{H}}=4.9 ;{ }^{3} J_{\mathrm{H}, \mathrm{H}}=2.1 ; 1 \mathrm{H}, \mathrm{N}-\mathrm{CH}_{2}\right), 5.42(\mathrm{~s}$, $1 \mathrm{H}, \mathrm{N}-\mathrm{CH}-\mathrm{N}), 7.10-7.20(\mathrm{~m}, 1 \mathrm{H}, \mathrm{Ph}), 7.30-7.45$ (m, 4H, Ph). ${ }^{13} \mathrm{C}$ NMR $\delta 8.7\left(\mathrm{H}_{3} \mathrm{C}-\mathrm{C}=\right), 22.0$ $\left(\mathrm{CH}_{3}\right), 22.6\left(=\mathrm{C}-\underline{\mathrm{CH}}_{2}\right), 25.5\left(\mathrm{CH}_{3}\right), 49.2\left(\mathrm{~N}-\mathrm{CH}_{2}\right), 55.8\left(\underline{\mathrm{C}}-\left(\mathrm{CH}_{3}\right)_{2}\right), 84.3(\mathrm{~N}-\mathrm{CH}-\mathrm{N}), 116.2\left(\mathrm{H}_{3} \mathrm{C}-\right.$ $\underline{\mathrm{C}}=), 122.8(\mathrm{~s}, 2 \times \mathrm{CH}, \mathrm{Ph}), 125.5(\mathrm{~s}, \mathrm{CH}, \mathrm{Ph}), 129.0(\mathrm{~s}, 2 \times \mathrm{CH}, \mathrm{Ph}), 139.2(\mathrm{C}, \mathrm{Ph}), 142.7$ (=-ㅡㄴ $\left.\mathrm{CH}_{2}\right), 162.3(\mathrm{C}=\mathrm{O})$.

(4S*, 9S*)-5,5,9-Trimethyl-3-phenyl-1,3,10-triazatricyclo[5.2.1 ${ }^{4,7} \cdot 0^{1,10}$ dec-6-en-2-one (8c). MP $118-119^{\circ} \mathrm{C}$. IR $\left(\mathrm{CHCl}_{3}\right) v_{\max } / \mathrm{cm}^{-1} 1227,1299,1380,1467,1499,1599,1711(\mathrm{C}=\mathrm{O})$, 2870, 2928, 2970. MS m/z (\%) 269 (M+; 100), 254 (84), 200 (15), 185 (25), 135 (97), 119 (22), 108 (39), 104 (57), 96 (50), 93 (29), 77 (68). HRMS calcd. for $\mathrm{C}_{16} \mathrm{H}_{20} \mathrm{~N}_{3} \mathrm{O}^{+}$270.1606; found 270.1595. ${ }^{1} \mathrm{H}$ NMR $\delta 0.80$ (s, 3H, $\left.\underline{\mathrm{H}}_{3} \mathrm{C}-\mathrm{C}-\mathrm{CH}_{3}\right), 1.36$ (s, 3H, $\left.\mathrm{H}_{3} \mathrm{C}-\mathrm{C}-\underline{\mathrm{C}}_{3}\right), 1.45$ (d, ${ }^{3} J_{\mathrm{H}, \mathrm{H}}=6.8$; $\left.3 \mathrm{H}, \mathrm{HC}-\underline{\mathrm{CH}}_{3}\right), 2.18\left(\mathrm{ddd},{ }^{2} J_{\mathrm{H}, \mathrm{H}}=15.5 ;{ }^{3} J_{\mathrm{H}, \mathrm{H}}=4.7 ;{ }^{4} J_{\mathrm{H}, \mathrm{H}}=2.4 ; 1 \mathrm{H}, \mathrm{CH}_{2}\right), 2.82\left(\mathrm{dd},{ }^{2} J_{\mathrm{H}, \mathrm{H}}=15.5 ;\right.$ $\left.{ }^{3} J_{\mathrm{H}, \mathrm{H}}=8.3 ; 1 \mathrm{H}, \mathrm{CH}_{2}\right), 4.38-4.50\left(\mathrm{~m}, 1 \mathrm{H}, \underline{\mathrm{HC}}-\mathrm{CH}_{3}\right), 4.66\left(\mathrm{dd},{ }^{4} J_{\mathrm{H}, \mathrm{H}}=2.4 ;{ }^{4} J_{\mathrm{H}, \mathrm{H}}=1.0 ; 1 \mathrm{H},=\mathrm{CH}\right)$, 5.39 (s, 1H, N-CH-N), $7.12-7.42(\mathrm{~m}, 5 \mathrm{H}, \mathrm{Ph}) .{ }^{13} \mathrm{C} \mathrm{NMR} \delta 23.4\left(\mathrm{CH}_{3}\right), 24.3\left(\mathrm{CH}_{3}\right), 27.4\left(\mathrm{CH}_{3}\right)$, $32.3\left(\mathrm{CH}_{2}\right), 54.9\left(\underline{\mathrm{C}}-\left(\mathrm{CH}_{3}\right)_{2}\right), 59.0\left(\mathrm{CH}_{2}-\underline{\mathrm{CH}}\right), 85.0(\mathrm{~N}-\mathrm{CH}-\mathrm{N}), 110.0(=\mathrm{CH}), 122.6(\mathrm{~s}, 2 \times \mathrm{CH}, \mathrm{Ph})$, 125.4 (s, CH, Ph), 129.1 (s, 2×CH, Ph), 139.1 (C, Ph), $150.2\left(=\underline{\mathrm{C}}-\mathrm{CH}_{2}\right), 161.8(\mathrm{C}=\mathrm{O})$. $\left(4 S^{*}, 9 S^{*}\right)-5,5,6,9-T e t r a m e t h y l-3-p h e n y l-1,3,10$-triazatricyclo $\left[5.2 .1^{4,7} \cdot 0^{1,10}\right]$ dec-6-en-2-one (7d). MP $112-113{ }^{\circ} \mathrm{C}$. IR $\left(\mathrm{CHCl}_{3}\right) v_{\max } / \mathrm{cm}^{-1} 1148,1243,1297,1380,1501,1598,1711$ $(\mathrm{C}=\mathrm{O}), 2869,2928,2968,3009 . \mathrm{MS} \mathrm{m} / \mathrm{z}(\%) 284\left(\mathrm{M}^{+}+1 ; 100\right), 269$ (38), 149 (50), 122 (26), 110 (18), 107 (19), 104 (19), 91 (19), 77 (35). HRMS calcd. for $\mathrm{C}_{17} \mathrm{H}_{22} \mathrm{~N}_{3} \mathrm{O}^{+}$284.1763; found 284.1758. ${ }^{1} \mathrm{H}$ NMR $\delta 0.81\left(\mathrm{~s}, 3 \mathrm{H}, \underline{\mathrm{H}}_{3} \mathrm{C}-\mathrm{C}-\mathrm{CH}_{3}\right), 1.37$ (s, 3H, $\left.\mathrm{H}_{3} \mathrm{C}-\mathrm{C}-\mathrm{C}_{3}\right), 1.53\left(\mathrm{~d},{ }^{3} J_{\mathrm{H}, \mathrm{H}}=6.8\right.$; $\left.3 \mathrm{H}, \mathrm{HC}-\underline{\mathrm{CH}}_{3}\right), 1.58\left(\mathrm{dd},{ }^{5} J_{\mathrm{H}, \mathrm{H}}=2.1 ;{ }^{5} J_{\mathrm{H}, \mathrm{H}}=0.7 ; 3 \mathrm{H}, \mathrm{H}_{3} \mathrm{C}-\mathrm{C}=\right), 2.09\left(\mathrm{ddm},{ }^{2} J_{\mathrm{H}, \mathrm{H}}=15.1 ;{ }^{3} J_{\mathrm{H}, \mathrm{H}}=\right.$ $\left.4.7 ; 1 \mathrm{H}, \mathrm{CH}_{2}\right), 2.81\left(\mathrm{dd},{ }^{2} J_{\mathrm{H}, \mathrm{H}}=15.1 ;{ }^{3} J_{\mathrm{H}, \mathrm{H}}=8.3 ; 1 \mathrm{H}, \mathrm{CH}_{2}\right), 4.35-4.46\left(\mathrm{~m}, 1 \mathrm{H}, \underline{\mathrm{HC}}-\mathrm{CH}_{3}\right), 5.42$ $(\mathrm{s}, 1 \mathrm{H}, \mathrm{N}-\mathrm{CH}-\mathrm{N}), 7.12-7.43(\mathrm{~m}, 5 \mathrm{H}, \mathrm{Ph}) .{ }^{13} \mathrm{C}$ NMR $\delta 8.6\left(\mathrm{H}_{3} \underline{\mathrm{C}}-\mathrm{C}=\right), 21.9\left(\mathrm{CH}_{3}\right), 23.3\left(\mathrm{CH}_{3}\right)$, 
$25.5\left(\mathrm{CH}_{3}\right), 31.0\left(\mathrm{CH}_{2}\right), 55.2\left(\underline{\mathrm{C}}-\left(\mathrm{CH}_{3}\right)_{2}\right), 58.4\left(\mathrm{CH}_{2}-\underline{\mathrm{CH}}\right), 84.5(\mathrm{~N}-\mathrm{CH}-\mathrm{N}), 115.7\left(\mathrm{H}_{3} \mathrm{C}-\underline{\mathrm{C}}=\right)$,

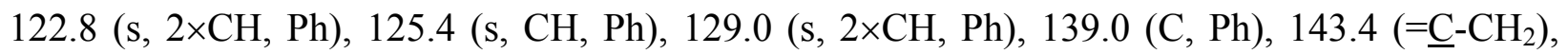
$162.1(\mathrm{C}=\mathrm{O})$.

$\left(4 S^{*}, \quad 9 S^{*}\right)-9-$ Ethyl-5,5-dimethyl-3-phenyl-1,3,10-triazatricyclo[5.2.1 $\left.{ }^{4,7} \cdot 0^{1,10}\right]$ dec-6-en-2-one (8e). IR (film) $v_{\max } / \mathrm{cm}^{-1} 1128,1149,1227,1300,1365,1383,1460,1500,1599,1718(\mathrm{C}=\mathrm{O})$, 2873, 2924, 2964. MS m/z (\%) 283 (M+; 100), 268 (46), 165 (47), 149 (27), 135 (19), 106 (27), 93 (36), 84 (88), 65 (19), 47 (65), 43 (37). ${ }^{1} \mathrm{H}$ NMR $\delta 0.78$ (s, 3H, $\left.\underline{\mathrm{H}}_{3} \mathrm{C}-\mathrm{C}-\mathrm{CH}_{3}\right), 1.02$ (t, ${ }^{3}$ $\left.J_{\mathrm{H}, \mathrm{H}}=7.3 ; 3 \mathrm{H}, \mathrm{CH}_{2}-\underline{\mathrm{CH}}_{3}\right), 1.33\left(\mathrm{~s}, 3 \mathrm{H}, \mathrm{H}_{3} \mathrm{C}-\mathrm{C}-\mathrm{CH}_{3}\right), 1.52-1.85$ (m, $\left.2 \mathrm{H}, \mathrm{CH}_{2}-\mathrm{CH}_{3}\right), 2.19$ (dm, $\left.{ }^{2} J_{\mathrm{H}, \mathrm{H}}=15.2 ; 1 \mathrm{H},=\mathrm{C}-\mathrm{CH}_{2}\right), 2.73\left(\mathrm{dd},{ }^{2} J_{\mathrm{H}, \mathrm{H}}=15.5 ;{ }^{3} J_{\mathrm{H}, \mathrm{H}}=8.3 ; 1 \mathrm{H},=\mathrm{C}-\mathrm{CH}_{2}\right), 4.04-4.20(\mathrm{~m}, 1 \mathrm{H}$, $\left.\underline{\mathrm{HC}}-\mathrm{CH}_{2}\right), 4.64$ (broad s, 1H, =CH), $5.38(\mathrm{~s}, 1 \mathrm{H}, \mathrm{N}-\mathrm{CH}-\mathrm{N}), 7.05-7.16(\mathrm{~m}, 1 \mathrm{H}, \mathrm{Ph}), 7.22-7.47$ (m, 4H, Ph). ${ }^{13} \mathrm{C}$ NMR $\delta 11.0\left(\mathrm{CH}_{2}-\underline{C H}_{3}\right), 24.1\left(\mathrm{CH}_{3}\right), 27.2\left(\mathrm{CH}_{3}\right), 29.9\left(\mathrm{CH}_{2}\right), 30.2\left(\mathrm{CH}_{2}\right), 54.7$ $\left(\mathrm{H}_{3} \mathrm{C}-\underline{\mathrm{C}}-\mathrm{CH}_{3}\right), 64.9\left(\mathrm{HC}-\mathrm{CH}_{2}\right), 84.6(\mathrm{~N}-\mathrm{CH}-\mathrm{N}), 109.7(=\mathrm{CH}), 122.1(\mathrm{~s}, 2 \times \mathrm{CH}, \mathrm{Ph}), 125.0(\mathrm{~s}, \mathrm{CH}$, $\mathrm{Ph}), 128.8$ (s, 2×CH, Ph), $139.0(\mathrm{C}, \mathrm{Ph}), 149.6\left(=\underline{\mathrm{C}}-\mathrm{CH}_{2}\right), 161.9(\mathrm{C}=\mathrm{O})$.

$\left(4 S^{*}, \quad 9 S^{*}\right)-9-E t h y l-5,5,6-t r i m e t h y l-3-p h e n y l-1,3,10$-triazatricyclo[5.2.1 $\left.{ }^{4,7} .0^{1,10}\right]$ dec-6-en-2one (8f). MP $114-115^{\circ} \mathrm{C}$. IR $\left(\mathrm{CHCl}_{3}\right) v_{\max } / \mathrm{cm}^{-1} 1149,1176,1240,1294,1367,1385,1394$, 1502, 1599, 1707 (C=O), 2877, 2937, 2968. MS m/z (\%) $297\left(\mathrm{M}^{+} ; 85\right), 282$ (62), 202 (35), 162 (22), 149 (100), 110 (32), 107 (24), 104 (24), 77 (42), 41 (15). HRMS calcd. for $\mathrm{C}_{18} \mathrm{H}_{24} \mathrm{~N}_{3} \mathrm{O}^{+}$ 298.1919; found 298.1927. ${ }^{1} \mathrm{H}$ NMR $\delta 0.74\left(\mathrm{~s}, 3 \mathrm{H}, \underline{\mathrm{H}}_{3} \mathrm{C}-\mathrm{C}-\mathrm{CH}_{3}\right), 1.04\left(\mathrm{t},{ }^{3} J_{\mathrm{H}, \mathrm{H}}=7.3 ; 3 \mathrm{H}, \mathrm{CH}_{2^{-}}\right.$ $\left.\underline{\mathrm{CH}}_{3}\right), 1.28\left(\mathrm{~s}, 3 \mathrm{H}, \mathrm{H}_{3} \mathrm{C}-\mathrm{C}-\mathrm{CH}_{3}\right), 1.49\left(\mathrm{dd},{ }^{5} J_{\mathrm{H}, \mathrm{H}}=1.8 ;{ }^{5} J_{\mathrm{H}, \mathrm{H}}=0.7 ; 3 \mathrm{H}, \mathrm{H}_{3} \mathrm{C}-\mathrm{C}=\right), 1.56-1.88(\mathrm{~m}$, $\left.2 \mathrm{H}, \underline{\mathrm{CH}}_{2}-\mathrm{CH}_{3}\right), 2.09\left(\mathrm{dm},{ }^{2} J_{\mathrm{H}, \mathrm{H}}=14.9 ; 1 \mathrm{H},=\mathrm{C}-\underline{\mathrm{H}}_{2}\right), 2.81\left(\mathrm{dd},{ }^{2} J_{\mathrm{H}, \mathrm{H}}=15.0 ;{ }^{3} J_{\mathrm{H}, \mathrm{H}}=8.3 ; 1 \mathrm{H},=\mathrm{C}-\right.$ $\left.\mathrm{C}_{2}\right), 4.05-4.18\left(\mathrm{~m}, 1 \mathrm{H}, \underline{\mathrm{HC}}-\mathrm{CH}_{2}\right), 5.42(\mathrm{~s}, 1 \mathrm{H}, \mathrm{N}-\mathrm{CH}-\mathrm{N}), 7.11-7.18(\mathrm{~m}, 1 \mathrm{H}, \mathrm{Ph}), 7.30-7.44$ $(\mathrm{m}, 4 \mathrm{H}, \mathrm{Ph}) .{ }^{13} \mathrm{C} \mathrm{NMR} \delta 8.6\left(\mathrm{H}_{3} \underline{\mathrm{C}}-\mathrm{C}=\right), 11.2\left(\mathrm{CH}_{2}-\mathrm{CH}_{3}\right), 22.0\left(\mathrm{CH}_{3}\right), 25.6\left(\mathrm{CH}_{3}\right), 29.3\left(\mathrm{CH}_{2}\right)$, $30.1\left(\mathrm{CH}_{2}\right), 55.4\left(\mathrm{H}_{3} \mathrm{C}-\underline{\mathrm{C}}-\mathrm{CH}_{3}\right), 64.7\left(\mathrm{HC}-\mathrm{CH}_{2}\right), 84.5(\mathrm{~N}-\mathrm{CH}-\mathrm{N}), 115.8\left(\mathrm{H}_{3} \mathrm{C}-\underline{\mathrm{C}}=\right), 122.6(\mathrm{~s}$, $2 \times \mathrm{CH}, \mathrm{Ph}), 125.3$ (s, CH, Ph), $129.0(\mathrm{~s}, 2 \times \mathrm{CH}, \mathrm{Ph}), 139.3(\mathrm{C}, \mathrm{Ph}), 143.3\left(=\underline{\mathrm{C}}-\mathrm{CH}_{2}\right), 162.5$ $(\mathrm{C}=\mathrm{O})$.

3-Benzyl-5,5-Dimethyl-1,3,10-triazatricyclo[5.2.1 $\left.{ }^{4,7} \cdot 0^{1,10}\right]$ dec-6-en-2-thione (8g). IR (film) $v_{\max } / \mathrm{cm}^{-1} 847,1049,1080,1128,1163,1227,1282,1308,1331,1385,1419,1446,1495,1682$, 2870, 2926, 2962. MS m/z (\%) 285 (M+1 100), 167 (37), 135 (25), 121 (32), 108 (50), 91 (87), 84 (88), 79 (15), 47 (31). ${ }^{1} \mathrm{H}$ NMR $\delta 1.21\left(\mathrm{~s}, 3 \mathrm{H}, \mathrm{CH}_{3}\right), 1.27\left(\mathrm{~s}, 3 \mathrm{H}, \mathrm{CH}_{3}\right), 2.46-2.61(\mathrm{~m}, 2 \mathrm{H},=\mathrm{C}-$ $\left.\mathrm{CH}_{2}\right), 3.70-3.89\left(\mathrm{~m}, 1 \mathrm{H}, \mathrm{N}-\mathrm{CH}_{2}\right), 4.26\left(\mathrm{~d},{ }^{2} \mathrm{~J}_{\mathrm{H}, \mathrm{H}}=15.2 ; 1 \mathrm{H}, \mathrm{CH}_{2}-\mathrm{Ph}\right), 4.43(\mathrm{~s}, 1 \mathrm{H}, \mathrm{N}-\mathrm{CH}-\mathrm{N})$, $4.54-4.71\left(\mathrm{~m}, 1 \mathrm{H}, \mathrm{N}-\mathrm{CH}_{2}\right), 4.69(\operatorname{broad} \mathrm{s}, 1 \mathrm{H},=\mathrm{CH}), 5.78\left(\mathrm{~d},{ }^{2} J_{\mathrm{H}, \mathrm{H}}=15.2 ; 1 \mathrm{H}, \mathrm{CH}_{2}-\mathrm{Ph}\right), 7.16-$ $7.43(\mathrm{~m}, 5 \mathrm{H}, \mathrm{Ph}) .{ }^{13} \mathrm{C}$ NMR $\delta 23.3\left(=\mathrm{C}-\mathrm{CH}_{2}\right), 23.7\left(\mathrm{CH}_{3}\right), 27.5\left(\mathrm{CH}_{3}\right), 51.0\left(\mathrm{CH}_{2}\right), 51.5\left(\mathrm{CH}_{2}\right)$, $55.7\left(\underline{\mathrm{C}}-\left(\mathrm{CH}_{3}\right)_{2}\right), 87.0(\mathrm{~N}-\mathrm{CH}-\mathrm{N}), 109.3(=\mathrm{CH}), 127.9(\mathrm{~s}, \mathrm{CH}, \mathrm{Ph}), 127.9(\mathrm{~s}, 2 \times \mathrm{CH}, \mathrm{Ph}), 128.7(\mathrm{~s}$, $2 \times \mathrm{CH}, \mathrm{Ph}), 134.7(\mathrm{C}, \mathrm{Ph}), 148.1$ (= $\left.\mathrm{C}-\mathrm{CH}_{2}\right), 187.7(\mathrm{C}=\mathrm{S})$.

5,5-Dimethyl-3-phenyl-1,3,10-triazatricyclo[5.2.1 $\left.{ }^{4,7} \cdot 0^{1,10}\right]$ dec-6-en-2-thione (8h). MP 166-169 ${ }^{\circ} \mathrm{C}$. IR $\left(\mathrm{CHCl}_{3}\right) v_{\max } / \mathrm{cm}^{-1} 1165,1257,1302,1340,1402,1458,1498,1597,1682,2868,2927$, 2966. MS m/z (\%) 271 (M+; 100), 256 (69), 185 (23), 136 (91), 121 (38), 108 (72), 104 (43), 93 (27), 77 (91), 67 (27), 41 (19). HRMS calcd. for $\mathrm{C}_{15} \mathrm{H}_{18} \mathrm{~N}_{3} \mathrm{~S}^{+}$272.1221; found 272.1194. ${ }^{1} \mathrm{H}$ NMR $\delta 0.77\left(\mathrm{~s}, 3 \mathrm{H}, \mathrm{CH}_{3}\right), 1.32\left(\mathrm{~s}, 3 \mathrm{H}, \mathrm{CH}_{3}\right), 2.53-2.73\left(\mathrm{~m}, 2 \mathrm{H},=\mathrm{C}-\mathrm{CH}_{2}\right), 3.82-4.00(\mathrm{~m}, 1 \mathrm{H}$, $\left.\mathrm{N}-\mathrm{CH}_{2}\right), 4.59-4.75\left(\mathrm{~m}, 1 \mathrm{H}, \mathrm{N}-\mathrm{CH}_{2}\right), 4.76$ (broad s, 1H, $\left.=\mathrm{CH}\right), 5.34(\mathrm{~s}, 1 \mathrm{H}, \mathrm{N}-\mathrm{CH}-\mathrm{N})$, 
$7.22-7.35(\mathrm{~m}, 1 \mathrm{H}, \mathrm{Ph}), 7.35-7.52(\mathrm{~m}, 4 \mathrm{H}, \mathrm{Ph}) .{ }^{13} \mathrm{C}$ NMR $\delta 23.7\left(=\mathrm{C}-\underline{C H}_{2}\right), 23.8\left(\mathrm{CH}_{3}\right), 28.0$ $\left(\mathrm{CH}_{3}\right), 53.0\left(\mathrm{~N}-\mathrm{CH}_{2}\right), 56.2\left(\underline{\mathrm{C}}-\left(\mathrm{CH}_{3}\right)_{2}\right), 90.0(\mathrm{~N}-\mathrm{CH}-\mathrm{N}), 109.9(=\mathrm{CH}), 126.9(\mathrm{~s}, 2 \times \mathrm{CH}, \mathrm{Ph}), 127.7$ (s, $\mathrm{CH}, \mathrm{Ph}), 129.0$ (s, 2×CH, Ph), $139.9(\mathrm{C}, \mathrm{Ph}), 148.1$ (= $\left.\underline{\mathrm{C}}-\mathrm{CH}_{2}\right), 187.6(\mathrm{C}=\mathrm{S})$.

5,5,6-Trimethyl-3-phenyl-1,3,10-triazatricyclo[5.2.1 $\left.{ }^{4,7} .^{1,10}\right]$ dec-6-en-2-thione (8i). MP 163 $164{ }^{\circ} \mathrm{C}$. IR $\left(\mathrm{CHCl}_{3}\right) v_{\max } / \mathrm{cm}^{-1} 1169,1257,1298,1342,1389,1404,1437,1456,1468,1498$, 1597, 1714, 2860, 2935, 2964. MS m/z (\%) 285 ( $\mathrm{M}^{+}$; 19), 270 (15), 150 (29), 135 (42), 122 (31), 108 (15), 77 (100), 65 (19), 51 (62), 41 (81). HRMS calcd. for $\mathrm{C}_{16} \mathrm{H}_{20} \mathrm{~N}_{3} \mathrm{~S}^{+}$286.1378; found 286.1364. ${ }^{1} \mathrm{H}$ NMR $\delta 0.69\left(\mathrm{~s}, 3 \mathrm{H}, \underline{\mathrm{H}}_{3} \mathrm{C}-\mathrm{C}-\mathrm{CH}_{3}\right), 1.25$ (s, 3H, $\left.\mathrm{H}_{3} \mathrm{C}-\mathrm{C}-\underline{\mathrm{H}}_{3}\right), 1.50$ (dd, ${ }^{5} J_{\mathrm{H}, \mathrm{H}}=2.0$; $\left.{ }^{5} J_{\mathrm{H}, \mathrm{H}}=0.7 ; 3 \mathrm{H}, \mathrm{H}_{3} \mathrm{C}-\mathrm{C}=\right), 2.42-2.70\left(\mathrm{~m}, 2 \mathrm{H},=\mathrm{C}-\mathrm{CH}_{2}\right), 3.64\left(\mathrm{ddd},{ }^{2} J_{\mathrm{H}, \mathrm{H}}=11.9 ;{ }^{3} J_{\mathrm{H}, \mathrm{H}}=5.7\right.$; $\left.{ }^{3} J_{\mathrm{H}, \mathrm{H}}=1.9 ; 1 \mathrm{H}, \mathrm{N}-\mathrm{CH}_{2}\right), 4.62\left(\mathrm{ddd},{ }^{2} J_{\mathrm{H}, \mathrm{H}}=9.0 ;{ }^{3} J_{\mathrm{H}, \mathrm{H}}=4.6 ;{ }^{3} J_{\mathrm{H}, \mathrm{H}}=1.7 ; 1 \mathrm{H}, \mathrm{N}-\mathrm{CH}_{2}\right), 5.35(\mathrm{~s}, 1 \mathrm{H}$, $\mathrm{N}-\mathrm{CH}-\mathrm{N}), 7.25-7.35(\mathrm{~m}, 1 \mathrm{H}, \mathrm{Ph}), 7.35-7.50(\mathrm{~m}, 4 \mathrm{H}, \mathrm{Ph}) .{ }^{13} \mathrm{C}$ NMR $\delta 8.4\left(\mathrm{H}_{3} \mathrm{C}-\mathrm{C}=\right), 21.6$ $\left(\mathrm{CH}_{3}\right), 22.6\left(=\mathrm{C}-\underline{\mathrm{CH}}_{2}\right), 26.2\left(\mathrm{CH}_{3}\right), 52.7\left(\mathrm{~N}-\mathrm{CH}_{2}\right), 56.5\left(\underline{\mathrm{C}}-\left(\mathrm{CH}_{3}\right)_{2}\right), 89.8(\mathrm{~N}-\mathrm{CH}-\mathrm{N}), 116.0\left(\mathrm{H}_{3} \mathrm{C}-\right.$ $\underline{\mathrm{C}}=), 127.1(\mathrm{~s}, 2 \times \mathrm{CH}, \mathrm{Ph}), 127.7(\mathrm{~s}, \mathrm{CH}, \mathrm{Ph}), 129.0(\mathrm{~s}, 2 \times \mathrm{CH}, \mathrm{Ph}), 140.1(\mathrm{C}, \mathrm{Ph}), 141.6(=\underline{\mathrm{C}}-$ $\left.\mathrm{CH}_{2}\right), 187.8(\mathrm{C}=\mathrm{S})$.

Dimethyl 9-isopropyl-5,5,6-trimethyl-1,10-diazatricyclo[5.2.1 $\left.{ }^{4,7} \cdot 0^{1,10}\right]$ dec-2,6-dien-2,3dicarboxylate (9). IR (film) $v_{\max } / \mathrm{cm}^{-1} 1128,1263,1348,1437,1608,1705(\mathrm{C}=\mathrm{O}), 1743(\mathrm{C}=\mathrm{O})$, 2872, 2956. GC-MS m/z (\%) $334\left(\mathrm{M}^{+}\right.$; 27), 207 (30), 153 (22), 107 (100), 94 (22). ${ }^{1} \mathrm{H}$ NMR $\delta$ $0.83\left(\mathrm{~d},{ }^{3} J_{\mathrm{H}, \mathrm{H}}=6.6 ; 3 \mathrm{H}, \mathrm{HC}-\left(\mathrm{C}_{3}\right)_{2}\right), 0.89\left(\mathrm{~d},{ }^{3} J_{\mathrm{H}, \mathrm{H}}=6.5 ; 3 \mathrm{H}, \mathrm{HC}-\left(\mathrm{CH}_{3}\right)_{2}\right), 0.96\left(\mathrm{~s}, 3 \mathrm{H}, \mathrm{CH}_{3}\right)$, $1.22\left(\mathrm{~s}, 3 \mathrm{H}, \mathrm{CH}_{3}\right), 1.47$ (s, 3H, $\left.\mathrm{CH}_{3}\right), 1.60-1.75$ (m, 1H, N-CH-C파), $2.10-2.30\left(\mathrm{~m}, 1 \mathrm{H}, \mathrm{CH}_{2}\right)$, $2.50-2.63\left(\mathrm{~m}, 1 \mathrm{H}, \mathrm{CH}_{2}\right), 3.32-3.42(\mathrm{~m}, 1 \mathrm{H}, \mathrm{N}-\mathrm{CH}-\mathrm{CH}), 3.61(\mathrm{~s}, 3 \mathrm{H}, \mathrm{OMe}), 3.80(\mathrm{~s}, 3 \mathrm{H}$, $\mathrm{OMe}), 4.31(\mathrm{~s}, 1 \mathrm{H}, \mathrm{HC}-\mathrm{C}=) .{ }^{13} \mathrm{C}$ NMR $\delta 8.3\left(\mathrm{H}_{3} \underline{\mathrm{C}}-\mathrm{C}=\right), 18.8\left(\mathrm{CH}_{3}\right), 19.4\left(\mathrm{CH}_{3}\right), 21.4\left(\mathrm{CH}_{3}\right), 27.7$ $\left(\mathrm{CH}_{2}\right), 27.7\left(\mathrm{CH}_{3}\right), 33.6(\mathrm{~N}-\mathrm{CH}-\underline{\mathrm{CH}}), 51.2(\mathrm{OMe}), 52.8(\mathrm{OMe}), 54.8\left(-\underline{\mathrm{C}}-\left(\mathrm{CH}_{3}\right)_{2}\right), 69.4(\mathrm{~N}-\underline{\mathrm{CH}}-$ $\mathrm{CH}), 76.5(\mathrm{H} \underline{\mathrm{C}}-\mathrm{C}=), 106.1 \quad(\underline{\mathrm{C}}-\mathrm{COOMe}), 115.8\left(\mathrm{H}_{3} \mathrm{C}-\underline{\mathrm{C}}=\right), 141.9 \quad\left(=\underline{\mathrm{C}}-\mathrm{CH}_{2}\right), 152.0 \quad(\mathrm{~N}-\underline{\mathrm{C}}-$ $\mathrm{COOMe}), 164.1(\mathrm{C}=\mathrm{O}), 164.3(\mathrm{C}=\mathrm{O})$.

\section{X-ray analysis data of $8 d$ and $8 f$}

The crystal structures of compounds $\mathbf{8 d}$ and $\mathbf{8 f}$ have been deposited at the Cambridge Crystallographic Data Centre and allocated the deposition numbers CCDC 720065 \& 720066.

\section{Acknowledgements}

Authors acknowledge Dr. Jan Taraba and Dr. Marek Nečas for the crystallographic measurements.

\section{References}

1. Krause, N.; Hashmi, A.; Stephen, K. Modern Allene Chemistry. Wiley-VCH: Weinheim, 2004, Vol.1. and 2. 
2. Zachová, H.; Man, S.; Nečas, M.; Potáček, M. Eur. J. Org. Chem. 2005, 12, 2548.

3. Potáček, M.; Marek, R.; Žák, Z.; Trottier, J.; Janoušek, Z.; Viehe, H. G. Tetrahedron Lett. 1993, 34, 8341.

4. Huisgen, R. Angew. Chem., Int. Ed. Engl. 1963, 2, 365.

5. Zachová, H.; Marek, R.; Man, S.; Taraba, J.; Potáček, M. Tetrahedron Lett. 2006, 47, 8157.

6. (a) Man, S.; Buchlovič, M.; Potáček, M. Tetrahedron Lett. 2006, 47, 6961. (b) Buchlovič, M.; Man, S.; Potáček, M. Tetrahedron 2008, 64, 42, 9953.

7. (a) Zwierzak, A.; Sulewska, A. Synthesis 1976, 835. (b) Chen, G. S.; Wilbur, J. K.; Barnes, Ch. L.; Glaser, R. J. Chem. Soc., Perkin Trans. 2 1995, 12, 2311.

8. Padwa, A. 1,3-Dipolar Cycloaddition Chemistry; Wiley: New York, 1984.

9. Gieren, A.; Narayanan, P.; Burger, K.; Thenn, W. Angew. Chem., Int. Ed. Engl. 1974, 13, 475.

10. Man, S.; Kulhánek, P.; Potáček, M.; Nečas, M. Tetrahedron Lett. 2002, 43, 6431.

11. Man, S.; Nečas, M.; Bouillon, J-P.; Baillia, H.; Harakat, D.; Potáček, M. Tetrahedron 2005, 61, 2387.

12. Schröter, H. B.; Neumann, D.; Katritzky, A. R.; Swinbourne, F.J. Tetrahedron 1966, 22, 8, 2895.

13. Ravikanth, V.; Ramesh, P.; Diwan, P. V.; Venkateswarlu, Y. Biochem. Syst. Ecol. 2001, 29, 753.

14. Adesanya, S. A.; Nia, R.; Fontaine, C.; Païs, M. Phytochemistry 1994, 35, 4, 1053.

15. Hueller, H.; Peters, R.; Scheler, W.; Schmidt, D.; Stremmel, D. Pharmazie 1971, $26,361$.

16. (a) Singh, B.; Saxena, A. K.; Chandan, B. K.; Gupta, D. K.; Bhutani, K. K.; Anand, K. K. Phytother. Res. 2001, 15, 311. (b) Davis, L.; Kuttan, G. J. Exp. Cancer. Res. 2002, $21,115$.

17. Kulhánek, P.; Koča, J.; Potáček, M. Collect. Czech. Chem. Commun. 2004, 69, 231.

18. Man, S.; Bouillon, J-P.; Nečas, M.; Potáček, M. Tetrahedron Lett. 2004, 45, 9419.

19. SHELXTL, Version 5.10, Bruker AXS Inc., Madison, WI, USA, 1997. 